\title{
On the Effect of Rigid Swept Surface Waves on Turbulent Drag
}

\author{
M. Denison* \\ National Institute of Aerospace, Hampton VA 23681, USA \\ S.P. Wilkinson ${ }^{\dagger}$ and P. Balakumar ${ }^{\ddagger}$ \\ NASA Langley Research Center, Hampton VA, 23681, USA
}

\begin{abstract}
Passive turbulent drag reduction techniques are of interest as a cost effective means to improve air vehicle fuel consumption. In the past, rigid surface waves slanted at an angle from the streamwise direction were deemed ineffective to reduce skin friction drag due to the pressure drag that they generate. A recent analysis seeking similarities to the spanwise shear stress generated by spatial Stokes layers suggested that there may be a range of wavelength, amplitude, and orientation in which the wavy surface would reduce turbulent drag. The present work explores, by experiments and Direct Numerical Simulations (DNS), the effect of swept wavy surfaces on skin friction and pressure drag. Plates with shallow and deep wave patterns were rapid-prototyped and tested using a drag balance in the $7 \times 11$ inch Low-Speed Wind Tunnel at the NASA LaRC Research Center. The measured drag offset between the wavy plates and the reference flat plate is found to be within the experimental repeatability limit. Oil vapor flow measurements indicate a mean spanwise flow over the deep waves. The turbulent flow in channels with flat walls, swept wavy walls and spatial Stokes spanwise velocity forcing was simulated at a friction Reynolds number of two hundred. The time-averaged and dynamic turbulent flow characteristics of the three channel types are compared. The drag obtained for the channel with shallow waves is slightly larger than for the flat channel, within the range of the experiments. In the case of the large waves, the simulation over predicts the drag. The shortcomings of the Stokes layer analogy model for the estimation of the spanwise shear stress and drag are discussed.
\end{abstract}

\section{Nomenclature}

$\Delta x \quad$ DNS Mesh cell size in the $\mathrm{x}$-direction

$\Delta y \quad$ DNS Mesh cell size in the y-direction

$\Delta z \quad$ DNS Mesh cell size in the z-direction

$\Delta z_{e} \quad$ Distance along the z-axis between the edge of the sub-domain with undamped sinusoidal wall profile and a point located on its outside

$\delta_{\theta} \quad$ Momentum thickness

$\delta_{v} \quad$ Friction length scale $\nu / u_{\tau}$

$\lambda \quad$ Wall wavelength in the direction perpendicular to the wave crests

$\lambda_{x} \quad$ Wall wavelength in the direction parallel to the $\mathrm{x}$-axis

$\lambda_{z} \quad$ Wall wavelength in the direction parallel to the z-axis

$\nu \quad$ Kinematic viscosity

$\sigma_{i, 3}(i=1,2,3)$ z-Components of the stress tensor on the faces of an infinitesimal volume perpendicular to the $\mathrm{x}, \mathrm{y}$ and $\mathrm{z}$ axes

$\tau_{z} \quad$ z-Component of the wall shear stress

*AIAA Senior Member.

${ }^{\dagger}$ Flow Physics and Control Branch, AIAA Senior Member.

${ }^{\ddagger}$ Flow Physics and Control Branch, AIAA Associate Fellow. 
$\widehat{\operatorname{var}}(\mathbf{x}, t, T)$ Localized variance

$\xi \quad$ Coordinate perpendicular to the wall wave crests

$A \quad$ Curve fit parameter for the drag coefficient $\left(C_{d}=A R e^{B}\right)$

$A_{S S L}$ Amplitude of the spanwise velocity oscillations at the wall for the Spatial Stokes layer

$B \quad$ Curve fit parameter for the drag coefficient $\left(C_{d}=A R e^{B}\right)$

$C_{d} \quad$ Total drag coefficient

$c_{f} \quad$ Friction coefficient

$c_{p} \quad$ Pressure coefficient

$D \quad$ Detection function for the local variance

$h \quad$ Channel half-height

$h_{w} \quad$ Wall wave amplitude

$k_{x} \quad$ Wall wavenumber in the direction parallel to the $\mathrm{x}$-axis

$k_{z} \quad$ Wall wavenumber in the direction parallel to the z-axis

$k_{t h} \quad$ Factor for the threshold of localized variance leading to an event detection

$L_{x} \quad$ Length of the simulation domain (in the x-direction)

$L_{z} \quad$ Width of the simulation domain (in the z-direction)

$M \quad$ Mach number of the freestream

$n_{i}(i=1,2,3) \mathrm{x}-, \mathrm{y}^{-}$, and $\mathrm{z}$-components of the unit vector perpendicular to the wall

$R \quad$ Ideal specific gas constant for air $(287 \mathrm{~J} /(\mathrm{kgK}))$

$R e / m$ Reynolds number per unit length $\left(u_{\infty} / \nu_{\infty}\right)$

$R e_{h} \quad$ Reynolds number defined as $u_{\infty} h / \nu_{\infty}$

$R e_{\tau} \quad$ Friction Reynolds number $\left(u_{\tau} h / \nu\right)$

$R e_{\theta} \quad$ Momentum thickness Reynolds number $\left(u_{\infty} \delta_{\theta} / \nu_{\infty}\right)$

$T$ Integration time for the calculation of the localized variance

$t \quad$ Time

$t_{0} \quad$ Detected time of maximum localized variance

$T_{\text {ref }} \quad$ Reference temperature $(300.67 \mathrm{~K})$ used in DNS

$u \quad$ Streamwise velocity component

$u^{\prime} \quad$ Streamwise velocity fluctuation with respect to the time-averaged streamwise velocity

$u_{r m s}^{\prime} \quad$ Root mean square of the streamwise velocity fluctuations

$u_{\infty} \quad$ Freestream velocity

$u_{\tau, \text { ref }}$ Shear velocity for the reference flat channel

$u_{\tau} \quad$ Shear velocity

$v \quad$ Vertical velocity component

$v^{\prime} \quad$ Vertical velocity fluctuation with respect to the time-averaged vertical velocity

$w \quad$ Spanwise velocity component

$x, y$ and $z$ Cartesian coordinates oriented in streamwise, vertical and spanwise directions, respectively

$y_{w} \quad$ Vertical coordinate of the bottom wall of the wavy channel $\left(y_{w}=h_{w} \sin \left(k_{x} x+k_{z} z\right)\right)$

\section{Introduction}

TURBUlENCE over air vehicles increases the skin friction drag that in turn drives up energy consumption 1 and combustion emissions. In parallel to active flow control and novel aerodynamic designs, passive Turbulent Drag Reduction (TDR) methods that rely on incremental infrastructure changes are desirable. Riblets and engineered surface roughness address this issue but still face challenges in terms of manufacturability, durability, and maintenance. Therefore, there is interest in investigating alternate or complementary passive techniques to reduce turbulent drag.

Spanwise traveling waves and surface oscillations were shown to be effective to reduce drag at low Reynolds number. ${ }^{1-5}$ Viotti $^{6}$ showed by Direct Numerical Simulation (DNS) of channel flows at a friction Reynolds number $R e_{\tau}=u_{\tau} h / \nu=200$ (where $u_{\tau}$ is the friction velocity of the reference flow, $h$ is the half-height of the channel, and $\nu$ is the kinematic viscocity) that in the near-wall region, the effect of steady streamwise oscillations of spanwise velocity forcing in the form of Spatial Stokes Layers (SSL) is similar to that of temporal oscillations of the wall in the spanwise direction. The net power savings from the SSL were found to be superior to the temporal forcing case, owing to an additional spanwise Reynolds stress production term 
of negative sign originating from the streamwise gradient of the spanwise velocity. ${ }^{7}$ Furthermore, Viotti $^{6}$ showed in selected cases that the scaled DNS phase-averaged turbulent spanwise SSL flow was identical to the laminar solution, and used a linear analysis to estimate the net power gain by spatial Stokes layers of small characteristic thickness. The practical realization of wall motion poses a number of challenges. ${ }^{5}$ Seeking a passive solution, Chernyshenko ${ }^{8}$ proposed to use swept wavy surfaces to generate steady spanwise shear stress oscillations. He considered such oscillations to be the dominant drag control element for the SSL. He concluded from a linear analysis that a small percentage of total drag reduction is possible for a passive swept wavy wall. At $R e_{\tau}=200$, maximum net power reduction was predicted for waves having a streamwise wavelength of about 1500 spatial wall units (defined as $\delta_{v}=\nu / u_{\tau}$ ), oriented at an angle of ca. $37^{\circ}$ from the mean stream, with a wave amplitude expected to generate an SSL-equivalent peak spanwise velocity of the order of 2 velocity wall units $\left(u_{\tau}\right)$. Swept surface waves were already proposed in the past. In Ref. [9, 10], Sengupta and Lekoudis proposed a reduced two-dimensional curvilinear turbulent boundary layer calculation to model the drag over rigid and traveling swept wall waves. The study indicated a slight improvement in skin friction drag with respect to the flat plate in a range of the wave angle, but it was over-compensated by the pressure drag.

The objective of the present work is to revisit the effect of rigid swept waves oriented at an angle from the freestream on turbulent drag, and specifically to explore the model proposed in Ref. [8]. To this purpose, plates with different patterns of waves were rapid-prototyped and tested using a direct drag balance in a low-speed wind tunnel. Three-dimensional Direct Numerical Simulations were conducted on channels with flat, wavy and spatial Stokes wall boundaries. Section II describes the wind tunnel tests. Section III provides a description of the simulation set-up and selected channel cases. Section IV reports the drag measurements and oil vapor flow test results. The simulation results are provided and discussed in Section V. Concluding remarks are given in Section VI.

\section{Experimental Set-Up}

\section{A. Tunnel and Drag Balance}

The experimental portion of the study was conducted in the NASA Langley Research Center (LaRC) 7x11 Inch Low Speed Wind Tunnel (7x11). The 7x11 tunnel is a closed-loop, fan-driven, unpressurized, lowspeed facility with a top speed of approximately $50 \mathrm{~m} / \mathrm{s}$. Broadband turbulence intensity in the freestream is approximately $0.5 \%$. Testing was conducted on the lower wall of the rectangular test section that was equipped with a single component, floating-element drag balance. The boundary layer on the lower wall of the test section was tripped to turbulence with a $0.91 \mathrm{~mm}$ diameter rod bonded to the contraction surface $578 \mathrm{~mm}$ upstream of the test model leading edge. Fully turbulent flow was observed for stream velocities greater than approximately $7 \mathrm{~m} / \mathrm{s}$. The useful unit Reynolds number range for the tunnel is approximately $0.5 \times 10^{6}<\operatorname{Re} / m<3.3 \times 10^{6}$. The momentum thickness Reynolds number range at the mid-point of the test section is approximately $750<R e_{\theta}<5200$.

The drag balance consists of a linear air bearing, a 0.25 Newton servo-type load cell, and associated hardware for leveling and positioning the test surface models. A schematic of the drag balance components, as situated beneath the test model, is shown in Fig. 1. The drag balance is fully mechanically isolated from the wind tunnel structure. This was primarily required as a means of preventing small, static tilt angles caused by tunnel structural distortion that would introduce drag measurement errors. While the drag balance is designed to measure horizontal forces, it is also very sensitive to inclination and the streamwise tilt angle must be tightly controlled. Tunnel frame distortion can be caused by aerodynamic loading within the tunnel loop or by thermally induced deflection of the tunnel structure due to ambient temperature variations or tunnel heating. The mechanical isolation also prevents tunnel vibrations from impacting drag balance performance. The air bearing system is basically an undamped spring-mass system with a characteristic frequency of about $15 \mathrm{~Hz}$. Without the mechanical isolation, the drag balance is subject to large oscillations at the resonant frequency rendering the drag data suspect. An earlier version of the system employed a 1/16inch $(1.59 \mathrm{~mm})$ thick rubber sheet to bridge the gap between the plenum chamber and support pedestal in place of the single pass, non-contact, labyrinth gland as currently shown in Fig. 1. The rubber sheet failed to prevent transmission tunnel vibrations and had to be removed. The labyrinth gland currently used has 
passage clearance of approximately 0.004 inch $(0.1 \mathrm{~mm})$.

The flotation gap around the model was designed as $0.01 \mathrm{inch}(0.25 \mathrm{~mm})$. The gap was checked after each model change and the model re-centered as required. The test model upper surface was maintained flush with the surrounding tunnel wall by use of leveling screws as shown in Fig. 1. The leading- and trailing-edge vertical misalignments were kept to 0.001 inch $(0.025 \mathrm{~mm})$ or better as measured with a dial indicator depth gage.

Air currents within the flotation gaps and within the plenum chamber are limiting factors in the ultimate accuracy and repeatability of the drag balance. The air bearing necessarily discharges air into the plenum that must be removed. Also, as the tunnel speed varies, the mean test section pressure drops causing a pressure difference between the plenum and test section. This difference causes flow in the flotation gaps leading to drag measurement errors. To prevent or minimize gap flow, the static pressure difference between the test section and plenum chamber was monitored with a 10 Torr full scale electronic pressure gage and adjusted to zero before each drag reading. Pressure equalization was accomplished by an external blower at constant rotational speed and a remotely controlled gate-type throttling valve. It was found necessary to introduce a small, fixed amount of external ambient air leakage into the plenum to allow the particular blower/gate-valve combination used in this study to operate effectively. For very low drag readings where the effects of dynamic pressure due to air currents in the plenum are commensurate with test model viscous forces, additional design improvements may be required, or, a different technique employed, such as elastic support flexures instead of the air bearing.

The air bearing itself has internal characteristics that can affect its performance. The particular air bearing used in the present investigation employs internal discharge orifices located in an offset fashion within machined depressions to distribute the air equally between the two exits. This allows the air bearing to operate "friction-free" overall but this characteristic is due to large equal and opposite internal viscous forces within the small clearance channels of the bearing. Any alteration of the internal equilibrium flow distribution not accounted for in the initial tare reading will cause drag measurement errors. Another type of air bearing commonly found uses internal porous metals or plastics for air distribution. In that case, the airflow pattern may be less predictable and even subject to different start-up flow patterns. The key to accurate use of air bearings for viscous drag measurements is to show that errors associated with air bearing design and operation are acceptable for the range of model forces being considered. The current design, as will be shown in Section IV, has 1-2\% repeatability. Improvement beyond this level requires additional study.

The streamwise test section pressure gradient due to boundary layer displacement thickness growth on the four upstream contraction walls and test section walls can also cause flow in the flotation gaps. The pressure gradient was approximately adjusted to zero by diverging the rear wall of the test section. An additional factor that could conceivably affect repeatability is the assumption that each time the tunnel is started, the turbulent boundary layer repeats exactly the same. Given the variability of boundary layer transition (only one wall was tripped in the current study), this is a tenuous assumption and may contribute to the $1-2 \%$ repeatability. Further study of this topic was beyond the scope of the current work.

\section{B. Test Models}

The flat plate and two wavy wall models were CNC-machined from aluminum using a commercial rapidprototype service provider. Photographs of the models are shown in Fig. 2. The wavy plates were sized following the guidance of Ref. [8]. The waves have a peak-to-valley amplitude of $2 h_{w}=232 \mu \mathrm{m}$ in one plate and $2 h_{w}=1045 \mu \mathrm{m}$ in the other. Below, these models will be referred to as the shallow waves and deep waves, with notations "WW232" and "WW1045", respectively. The sweep angle of the waves measured from the axial (x) coordinate is $37.4^{\circ}$. The wavelength $\lambda$ in the direction perpendicular to the wave crests is 26.8 $\mathrm{mm}$ for each case, giving aspect ratios of $h_{w} / \lambda=0.43$ and $1.95 \%$. The waves are damped within one wavelength at the plate periphery, as shown in Fig. 3(b). The net volume of the wavy plates is identical to that of the flat plate. The wetted area is increased by $0.013 \%$ for the shallow waves and $0.27 \%$ for the deeper waves.

The surface of each model was lightly glass-beaded at the service provider to remove CNC-machining marks and subsequently polished at LaRC prior to drag testing. Using a dilute detergent solution as a 


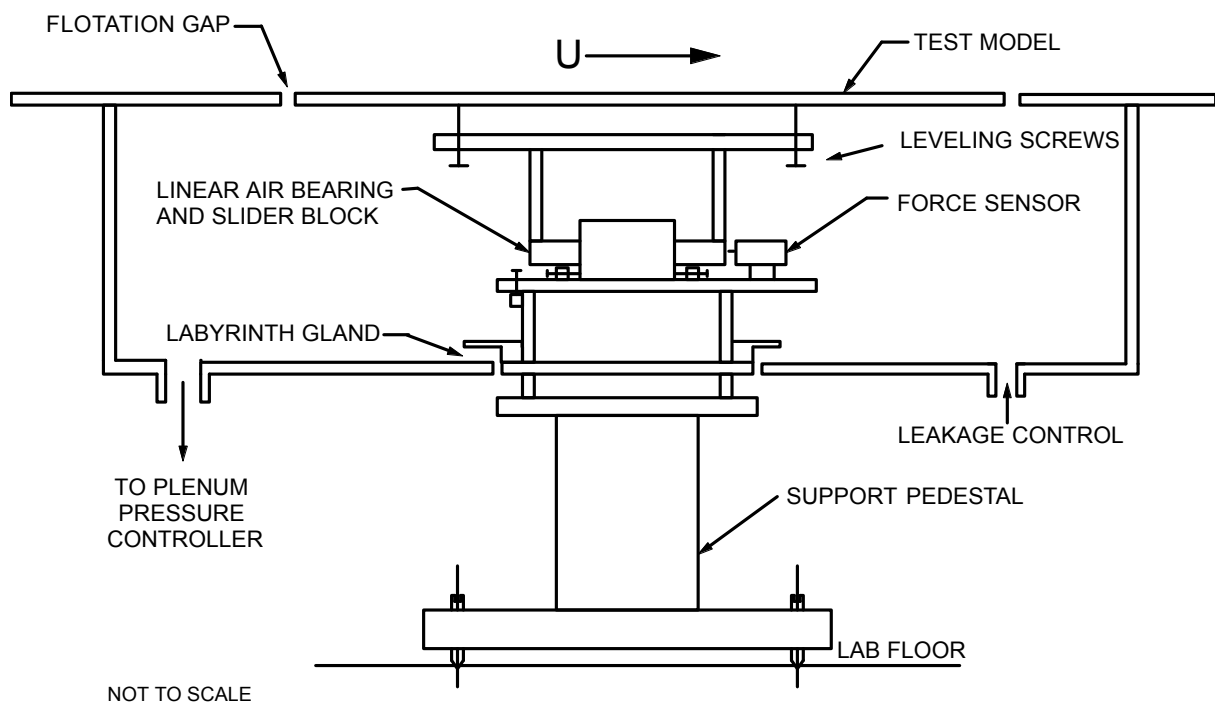

Figure 1: Schematic representation of floating drag balance components (for illustration only - not to scale).

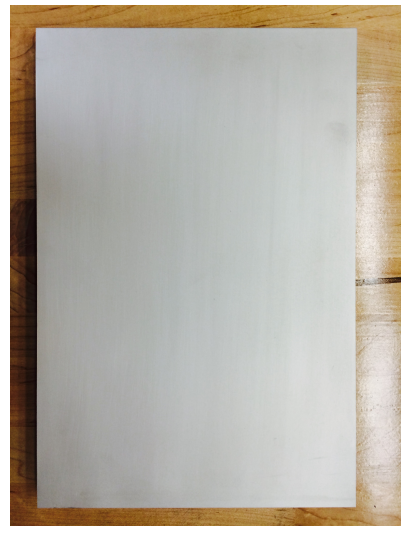

(a) Flat plate.

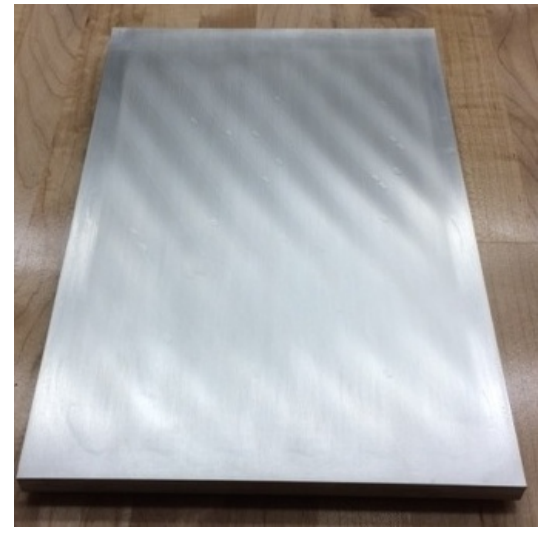

(b) Wavy plate with $h_{w} / \lambda=0.43 \%$.

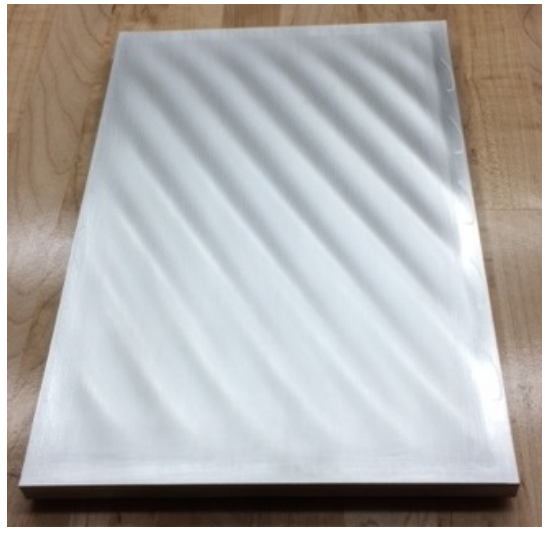

(c) Wavy plate with $h_{w} / \lambda=1.95 \%$.

Figure 2: Test plates. 


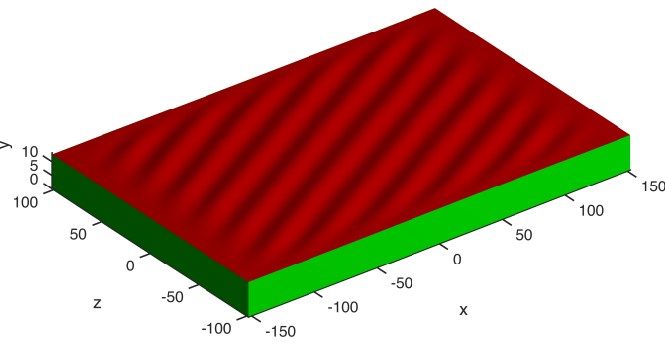

(a) Plate geometry.

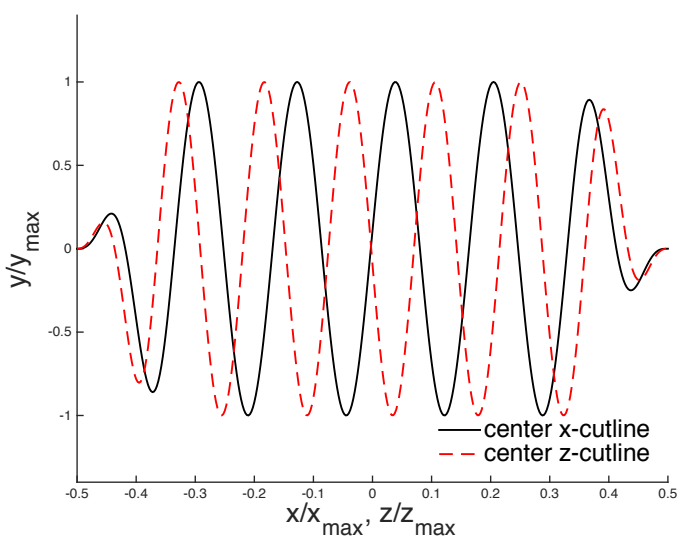

(b) Normalized surface cutlines through the plate center.

Figure 3: Plate with swept surface waviness.

lubricant, the models were first lightly sanded with medium abrasive, conformal foam pads followed by hand polishing 600-grit wet carbide sandpaper. The peak-to-valley wave height of each model was sampled with a dial-indicating depth gage and found to be within $2 \%$ of the design values.

Data acquisition was accomplished using a digital oscilloscope with 12-bit effective resolution (Agilent Model MSO-7034A in high resolution mode). The force sensor output was low-pass filtered at $0.1 \mathrm{~Hz}$, $12 \mathrm{~dB} /$ octave, and unity gain. The sensor output was sampled at $5 \mathrm{~Hz}$ and averaged over one 50 -second oscilloscope trace. The range of forces encountered varied from about 0.02 to $0.2 \mathrm{~N}$ on models with a plan area of $0.062 \mathrm{~m}^{2}$ for an average shear stress range of 0.32 to $3.2 \mathrm{~Pa}$.

Oil vapor flow visualization tests (i.e., the "smoke-wire" method) were performed to check for evidence of spanwise deviation of the mean flow over the waves. Prior to the oil test, the plates were painted with flat black lacquer to increase the contrast of the oil vapor over the aluminum surface. The oil was lightweight mineral oil. The stainless steel (type 304V) smoke wire had a diameter of $0.13 \mathrm{~mm}$ and was situated $7 \mathrm{~mm}$ downstream of the model leading edge, approximately $0.5 \mathrm{~mm}$ above the surface. Power was provided by an adjustable DC power supply and manually switched through a solid state relay. The vapor was side-lit with a cold fiber optic light source. Imaging was accomplished by full frame digital video sequences.

\section{CFD Modeling}

Three-dimensional channel Direct Numerical Simulations (DNS) were conducted using a compressible fifth order Weighted Essentially Non-Oscillatory (WENO) code for spatial discretization, with third order TotalVariation-Diminishing (TVD) scheme for temporal integration. ${ }^{11-13}$ The mesh size is $1224 \times 257 \times 416$ in the $\mathrm{x}, \mathrm{y}$, and $\mathrm{z}$ directions, respectively, with the $\mathrm{x}$-axis parallel to the inflow direction and the $\mathrm{y}$-axis oriented in the vertical direction. The mesh is uniform in $\mathrm{x}$ and $\mathrm{z}$ directions and is refined at the walls using a Roberts' transformation. ${ }^{14}$ In the flat channel the cell size along the walls is such that $\Delta x^{+}<2.8, \Delta y^{+}<0.18$, and $\Delta z^{+}<5.8$, where the + superscript indicates wall units. At the channel center, $\Delta y^{+}<3.5$.

Five channel cases are studied at $R e_{\tau}=200$, with the following wall boundaries: (1) flat walls with non-slip boundary conditions (reference), (2-3) flat walls with spanwise velocity forcing $W=A_{S S L} \sin \left(k_{x} x\right)$, where $A_{S S L} / u_{\tau, r e f}=2$ and 6 , and (4-5) walls with slanted waves defined by $y_{w}=h_{w} \sin \left(k_{x} x+k_{z} z\right)(+2 h)$ with two amplitude-to-wavelength ratios, $h_{w} / \lambda=0.05$ and $h_{w} / \lambda=2.25 \%$. The latter amplitudes correspond to the SSL velocity forcing amplitudes $A_{S S L} / u_{\tau, r e f}=2$ and 9 per the model proposed in Ref. [8]. Table 1 summarizes key simulation parameters. The Reynolds number based on the channel half-height and freestream velocity is $R e_{h}=3200 . u_{\tau, r e f}$ is the reference flat plate skin friction velocity that was used a-priori to size the wavy wall and Stokes problems. The notation $A_{S S L}^{+}$refers to the SSL wall velocity 
Table 1: DNS Parametrization

\begin{tabular}{lc}
\hline \hline Common parameters & \\
\hline$R e_{\tau}$ & 200 \\
$R e_{h}$ & 3200 \\
$M$ & 0.3 \\
$u_{\tau, r e f}$ & 0.062 \\
\hline Wavy wall & \\
\hline$\lambda_{x}^{+}$ & 1520 \\
$k_{x} / k_{z}$ & 0.7657 \\
$A_{S S L} / u_{\tau, r e f}$ & 2,9 \\
$h_{w} / \lambda$ & $0.5,2.25 \%$ \\
$\lambda_{x} / h$ & 7.6 \\
$L_{x} / \lambda_{x}, L_{z} / \lambda_{z}$ & 2 \\
\hline Spatial Stokes Layer & \\
\hline$\lambda_{x}^{+}$ & 1520 \\
$A_{S S L} / u_{\tau, r e f}$ & 2,6 \\
$A_{S S L}^{+}\left(\right.$actual $\left.u_{\tau}\right)$ & $2.17,7.65$ \\
\hline \hline
\end{tabular}

oscillation amplitude non-dimensionalized by the SSL skin friction velocity obtained from DNS. The domain size is common to all cases and was chosen to comprise two periods of the wavy wall in each direction, as shown in Fig. 4(a). There is no phase shift between the top and bottom wall profiles of the wavy channels, to avoid mean flow deviations from a varying cross-section.

Periodic boundary conditions are applied in the stream- and span-wise directions. The flow is maintained by applying a body force in the streamwise direction, determined at every step of the time marching by requiring that the average mass-flux remains constant. Depending on the wave amplitude, there may be a net spanwise component of the mean flow over the wavy wall. In absence of z-directed body force, such cross-flow may cause a spanwise pressure gradient affecting the streamwise drag. To assess the effect of the boundary conditions, two additional cases were simulated, with only one wavelength of wavy surface in spanwise direction, smoothly transitioning to flat edges towards $z=0$ and $z=L_{z}$. Such a channel is shown in Fig. 4(b). The transition is achieved by multiplying the channel profile by a $\cos ^{2}\left(k_{z} \Delta z_{e}\right)$ factor at its periphery, where $\Delta z_{e}$ is the distance along the z-axis between the edge of the region with undamped sinusoidal wall profile and a point located on its outside.

\section{Experimental results}

Fig. 5 shows the average of two drag data sets for the models WW232, WW1045, and the reference smooth flat plate that were acquired over two consecutive days to account for any day-to-day variations in drag. The solid curves are least square power laws of the form $C_{d}=A R_{e}^{B}$ where $C_{d}$ is the total drag coefficient and Re is the unit Reynolds number per meter. Curve fit coefficients are listed in Table 1. Fig. 5 shows little, if any, significant difference between the reference flat plate and the two wavy wall models.

The power law curve fit permits closer examination of the drag data relative to the smooth flat plate. This is shown in Fig. 6, where the percent difference between the wavy walls and flat plate is plotted based on the curve fits in Table 1. The zero value on the vertical axis represents the two-day flat plate average in Table 2. The figure shows that the day-to-day non-repeatability for each model's drag is at about the same level as drag differences between the models. For this reason, the small drag differences are not considered significant and within this 1-2\% band of uncertainty, no definitive statements regarding the drag increase 


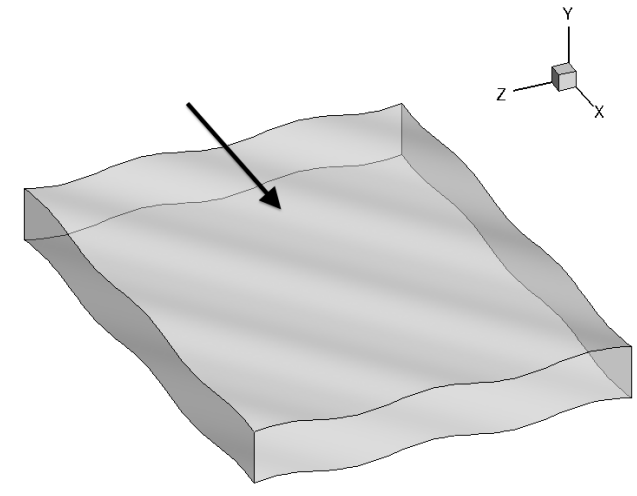

(a) Channel with wavy surface extending over the full xz-domain of area $2 \lambda_{x} \times 2 \lambda_{z}$.

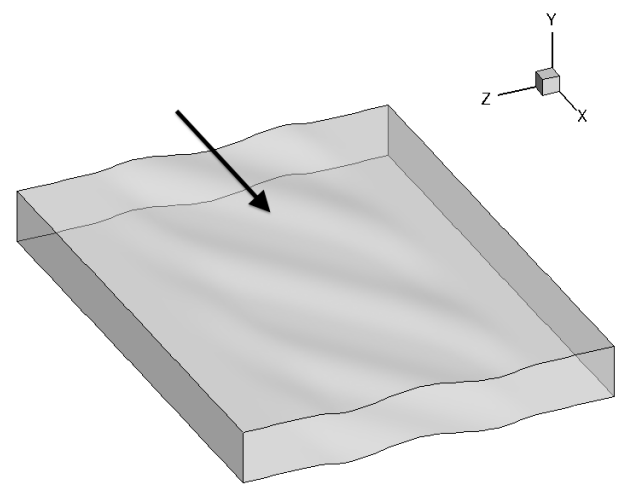

(b) Channel with center wavy surface of area $2 \lambda_{x} \times$ $1 \lambda_{z}$. The waves are damped towards the flat boundaries at $z=0$ and $z=L_{z}$

Figure 4: Physical simulation domains for the wavy channels. The black arrow indicates the direction of the inflow.

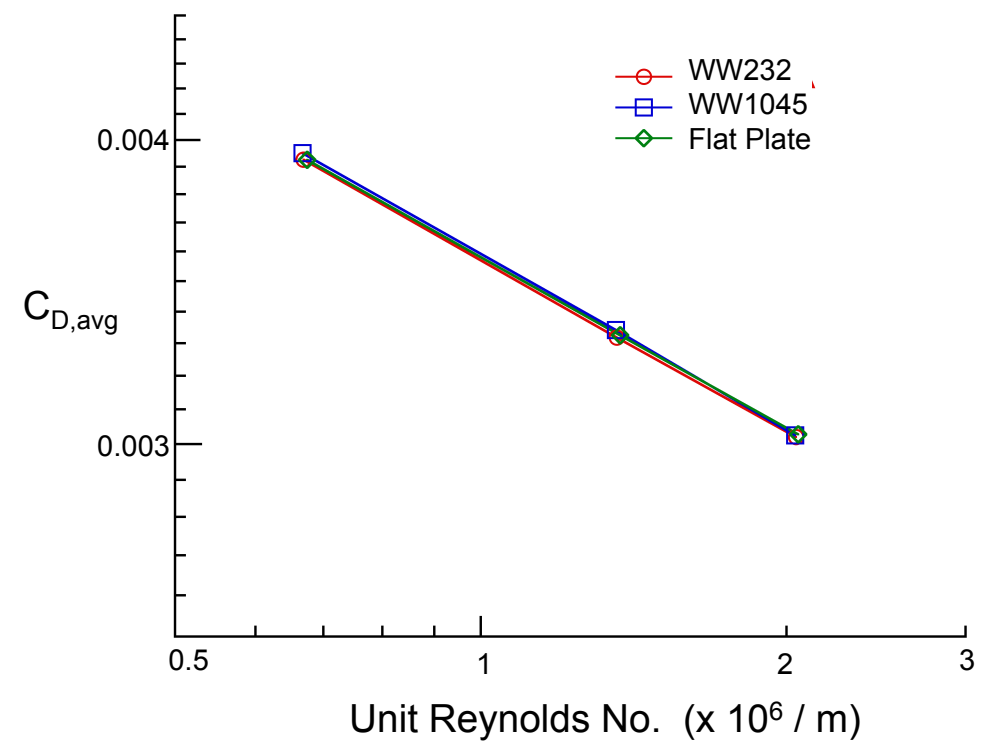

Figure 5: Average total drag coefficient with respect to the unit Reynolds number. 
or decrease of the wavy wall models can be made. The increased uncertainty at lower Reynolds numbers is consistent with the discussion of drag balance error sources in Section II. The data show, however, that the wavy walls, especially the large amplitude WW1045 model, do not increase the drag beyond this 1-2\% uncertainty level.

Table 2: Power law curve fit parameters, $C_{d}=A R_{e}^{B}$

\begin{tabular}{lccc}
\hline \hline Model & Day & A & B \\
\hline Flat plate & 1 & 0.109 & -0.247 \\
$"$ & 2 & 0.074 & -0.220 \\
$"$ & 2-day average & 0.092 & -0.234 \\
WW232 & 1 & 0.100 & -0.240 \\
$"$ & 2 & 0.086 & -0.231 \\
$"$ & 2-day average & 0.093 & -0.236 \\
WW1045 & 1 & 0.088 & -0.232 \\
$"$ & 2 & 0.108 & -0.247 \\
$"$ & 2-day average & 0.098 & -0.240 \\
\hline
\end{tabular}

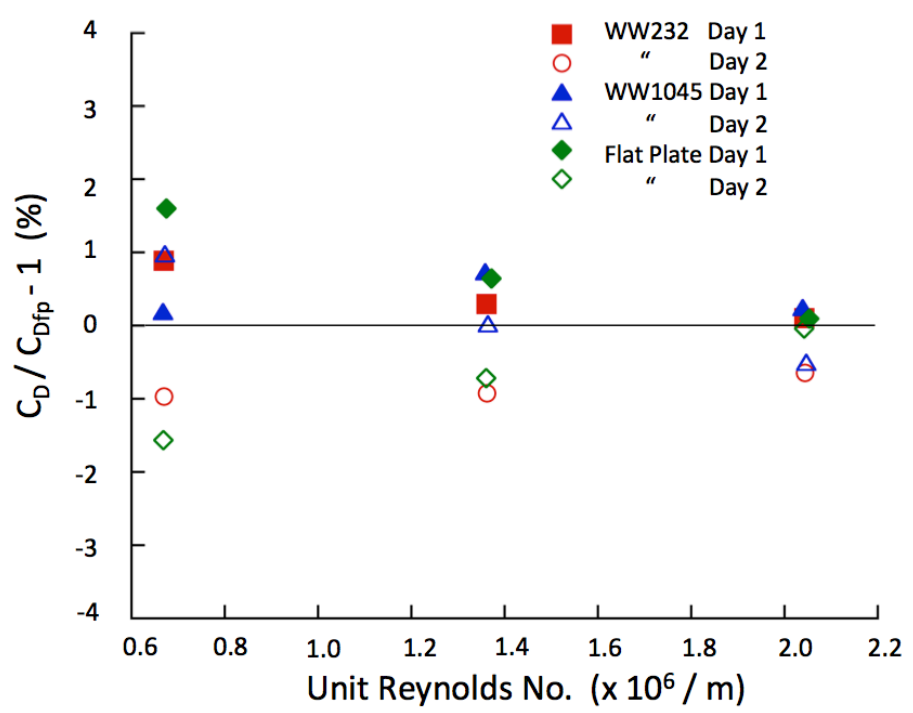

Figure 6: Drag coefficients relative to the smooth flat plat based on power law curve fits.

\section{A. Oil vapor flow test}

Oil vapor flow visualization tests were performed to check for any spanwise deviation of the mean flow over the waves. Fig. 7 shows photographs of oil vapor flow tests. In Fig. 7(a), the amplitude of the flow oscillations over the plate with $h / \lambda=0.43 \%$ is very small. The mean flow remains mostly two-dimensional, with no noticeable spanwise component. In contrast, the streaklines over the plate with $h / \lambda=1.95 \%$ in Fig. 7 (b) show large spanwise oscillations indicative of the non-linearity of the disturbance induced by the waves. There is also a net spanwise mean flow, as for example indicated by the deviation of the wave highlighted in Fig. 7(c) from the direction of the inflow. The deviation angle is found to vary within $2.5^{ \pm} 1.5^{\circ}$. These results support the assumption made at the z-periodic boundaries for the shallow waves. For the deep waves, the validity of the periodic conditions used remains questionable. 

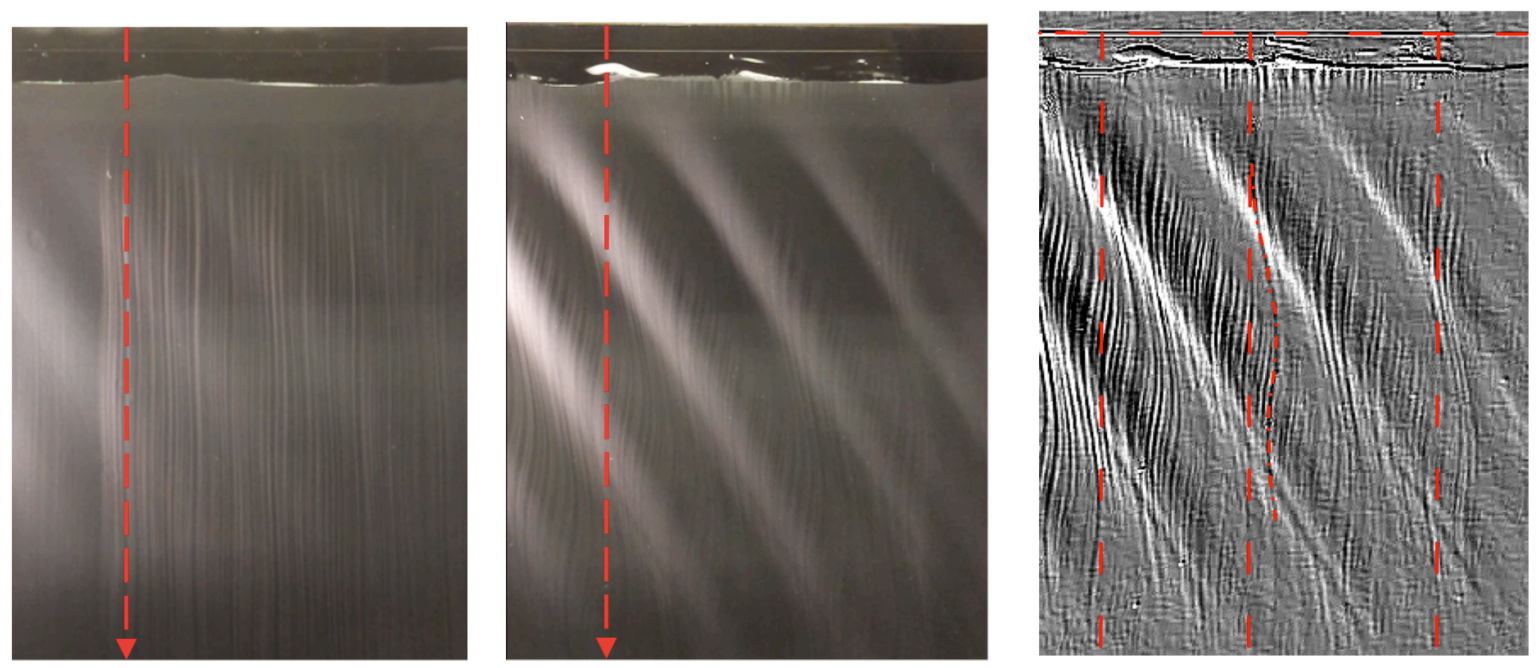

(a) Wavy plate with $h_{w} / \lambda=0.43 \%$. (b) Wavy plate with $h_{w} / \lambda=1.95 \%$. (c) Wavy plate with $h_{w} / \lambda=1.95 \%$ (emThe red arrow indicates the direction The red arrow indicates the direction of bossed and sharpened version of the image of the inflow. in (b)). The dash-dot line highlights a particular streakline. The horizontal dashed line shows the position of the wire. The three parallel dashed lines indicate the direction of the inflow.

Figure 7: Oil vapor flow test.

\section{CFD Analysis}

\section{A. Average turbulent flow}

Fig. 8 shows streamlines of time-averaged velocity at the surface of the bottom wall and in the center horizontal plane $(y=h / 2)$ of the wavy channel with $h_{w} / \lambda=0.05 \%$. A mean flow deviation of less than $0.1^{\circ}$ from the $\mathrm{x}$-axis (towards negative $\mathrm{z}$ ) is observed. Although not shown here, the same observation is made for the domain with flat edges per Fig. 4(b). This minor deviation is of higher order than the oscillatory flow perturbation, and is not expected to affect the drag analysis. In the case of the large waves with $h_{w} / \lambda=2.25 \%$, Fig. 9(a) reveals flow along the wave troughs. At the bottom wall, the convergence of streamlines along the downhill slope of the waves, and their divergence back to a locally parallel flow over the uphill slope is indicative of flow lift-up and lift-down, respectively. This is illustrated in the streamwise velocity profiles of Fig. 11. A flow along the wave troughs is also observed in the wavy area of the domain with flat edges, Fig. 10(a). Additionally, Figures 9-10(b) show that there is a net spanwise mean flow component. The transition length of $\lambda_{z} / 2$ from waves to flat edges used in the domain of Fig. 4(b) is too short to allow the flow to realign along the domain edges. For both partially and fully wavy domains, the angle formed by the streamlines with the $\mathrm{x}$-axis at mid-channel height is about $2.6^{\circ}$, which is within the range of the oil vapor flow measurements for $h_{w} / \lambda=1.95 \%$.

The average skin friction coefficient of the flat channel was computed as $c_{f}=7.46 \times 10^{-3}$, which is in reasonable agreement with the empirical expectation $c_{f}=\frac{0.0706}{\left(2 R e_{h}\right)^{1 / 4}}=7.89 \times 10^{-3} \cdot{ }^{15,16}$ Table 3 summarizes the drag results obtained for the different test cases after a minimum of 5 flow passages across the channel, normalized by the flat channel data. In this table, a positive drag reduction means that the drag is reduced. The drag in the SSL channel with $A_{S S L}^{+}=7.65$ is $33.9 \%$ lower than for the flat channel, which compares well with the TDR of $32 \%$ obtained at $\lambda_{x}=1250$ in Ref. [6]. The SSL channel with $A_{S S L}^{+}=2.17$ yields $8.5 \%$ drag reduction. The wavy wall leads to $0.3 \%$ skin friction increase and $1.1 \%$ pressure drag, yielding $1.4 \%$ total drag increase. This result in within the experimental resolution window of $2 \%$ reported in Section IV. The skin friction drag increase for the deep waves is found to be $2.7 \%$, with an excessive pressure drag that is still being analyzed in the light of the mean flow drift observations. The drag data obtained from the 


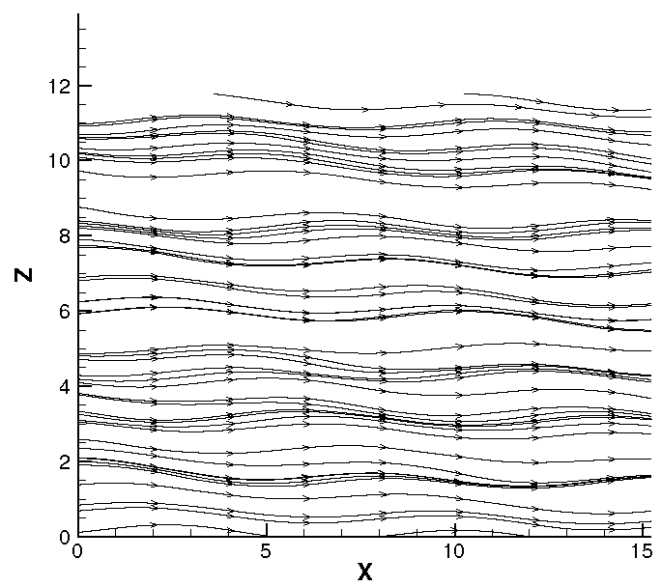

(a) Lower wall surface.

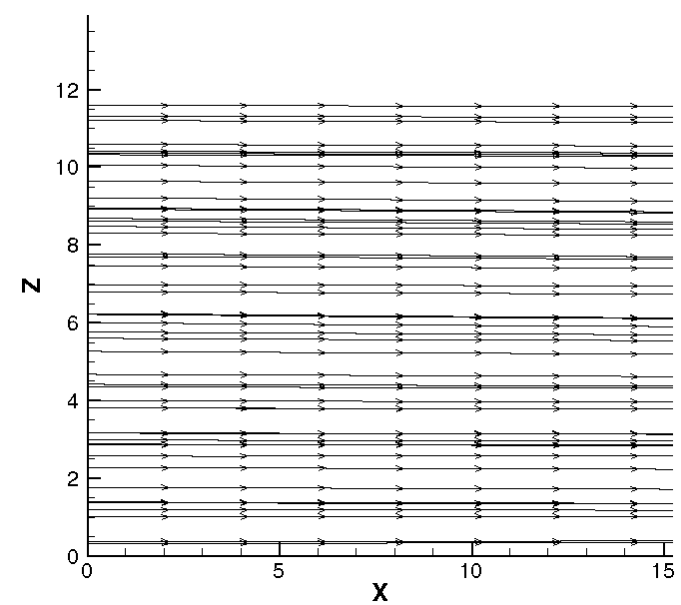

(b) Channel center $(y=h)$.

Figure 8: Streamlines of average velocity in the wavy channel with $h_{w} / \lambda=0.05 \%$. The flow goes from left to right.

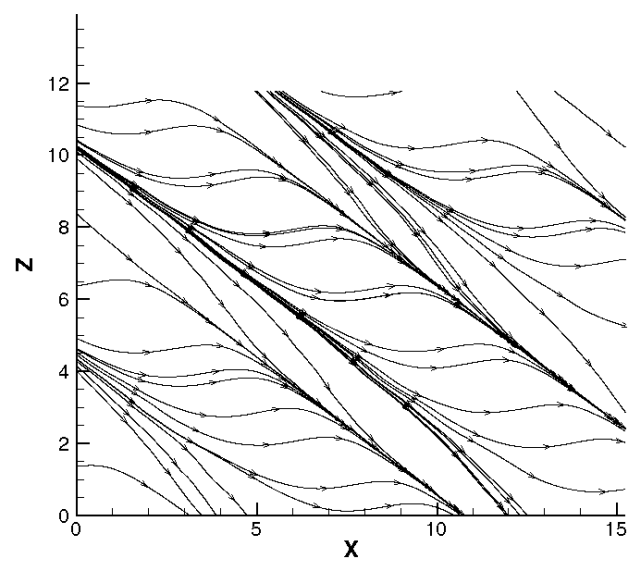

(a) Lower wall surface.

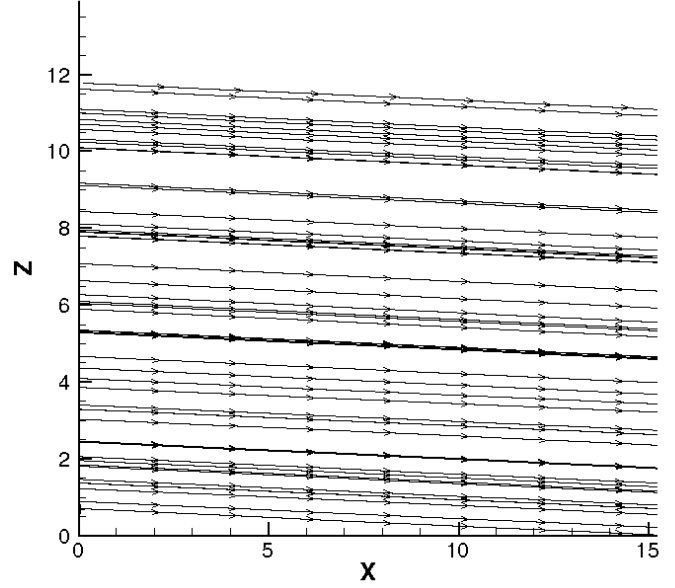

(b) Channel center $(y=h)$.

Figure 9: Streamlines of average velocity in the wavy channel with $h_{w} / \lambda=2.25 \%$. The flow goes from left to right. 


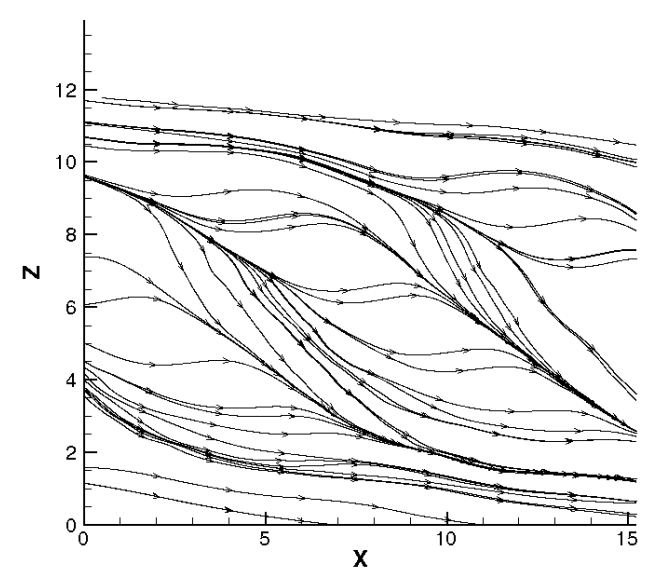

(a) Lower wall surface.

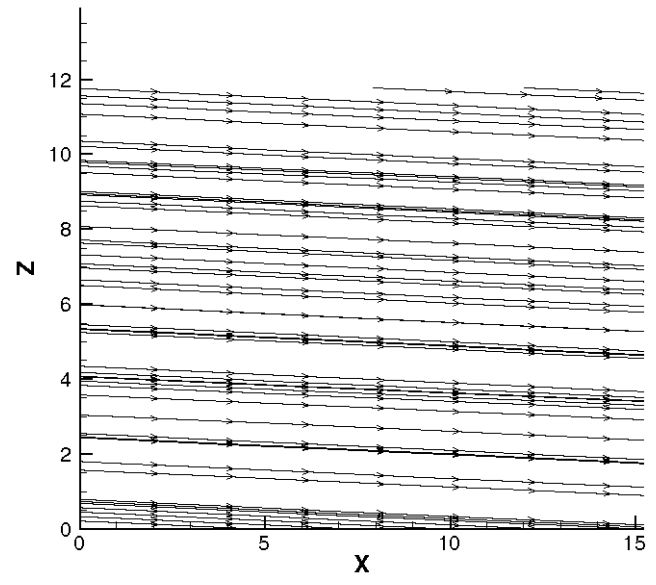

(b) Channel center $(y=h)$.

Figure 10: Streamlines of average velocity in the wavy channel with $h_{w} / \lambda=2.25 \%$ and flat edges at $z=0$ and $z=L_{z}$. The flow goes from left to right.

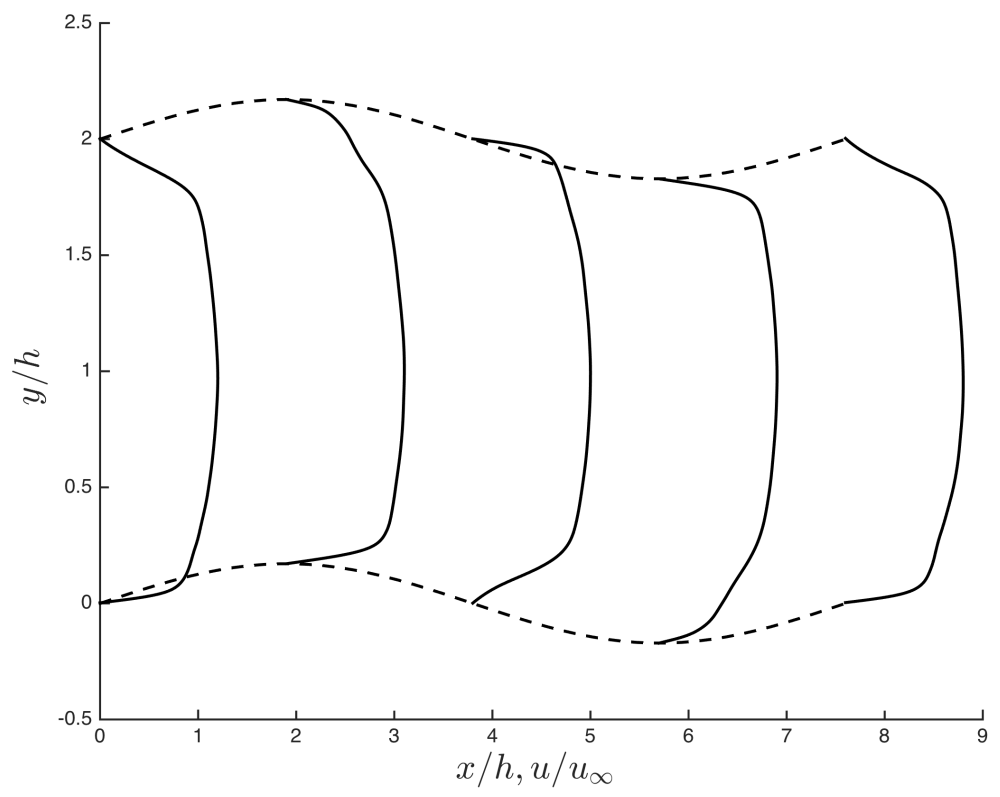

Figure 11: Streamwise velocity profiles (solid lines) at different positions along the wavy channel (of which the boundaries are shown by dashed lines) with $h_{w} / \lambda=2.25 \%$ at $z=0$. 
wavy domains with flat edges at $z=0$ and $z=L_{z}$ compare to those for the fully wavy domains. Neither the wind tunnel tests nor the DNS models were able to demonstrate turbulent drag reduction for the selected wall and flow parameters.

Table 3: Drag with respect to the flat channel

\begin{tabular}{lccc}
\hline \hline Drag component & SSL $A^{+}=7.65$ & SSL $A^{+}=2.17$ & $h_{w} / \lambda=0.05 \%$ \\
\hline \% Friction drag & 66.1 & 91.4 & 100.3 \\
\% Pressure drag & - & - & 1.1 \\
\% Drag reduction & 33.9 & 8.6 & -1.4 \\
\hline
\end{tabular}
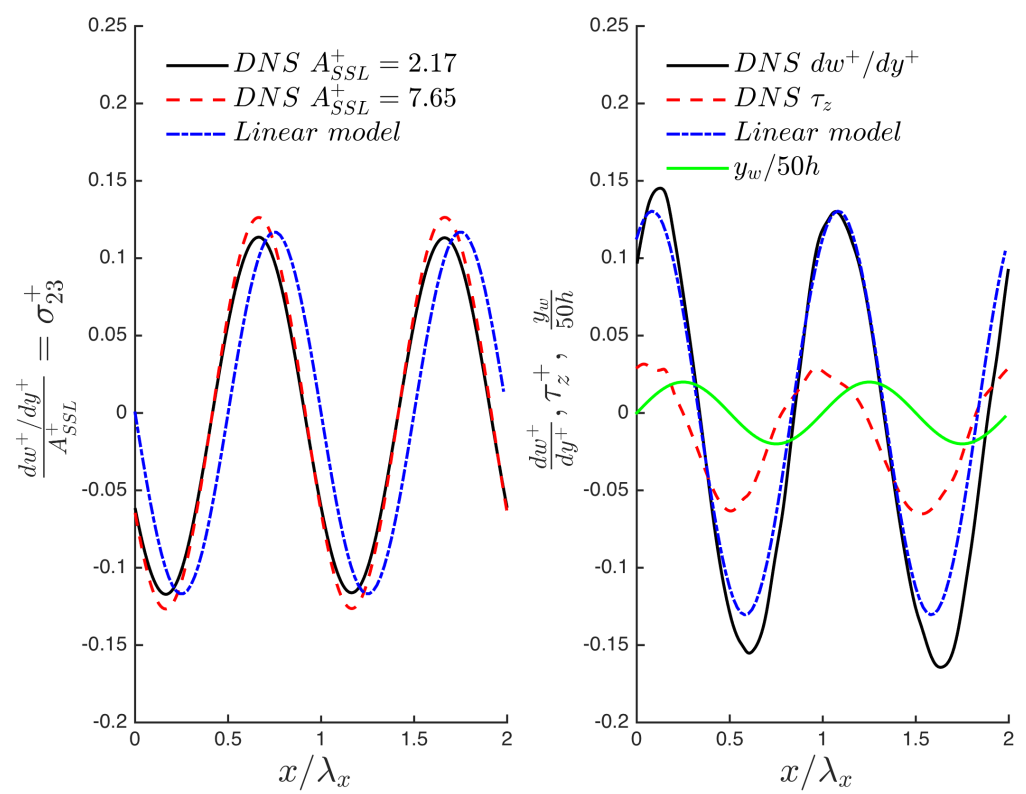

Figure 12: Comparison of the surface shear stress computed by DNS at $z=\lambda_{z}$ to that predicted by linear theory, in the spatial Stokes layer (LHS) and in the wavy wall (RHS). The wavy wall profile $y_{w}$ scaled by a factor of $1 / 50 h$ is shown in the right panel.

In earlier work, a good agreement was reported between the spanwise wall shear stress simulated by DNS in spatial Stokes layers, and that predicted by the linear model presented in Ref. [6,7]. The left panel of Fig. 12 compares the spanwise shear stress per unit of spanwise forcing predicted by this model, to that obtained by the DNS of the two SSL layers considered here. The agreement on the amplitude is within $0.5 \%$ for $A_{S S L}^{+}=2.17$ and $8.5 \%$ for $A_{S S L}^{+}=7.65$. The linear model proposed in Ref. [8] for the wall shear stress was based on a thin boundary layer assumption with a pressure perturbation of the same wave number as the wall, and no-slip boundary conditions at the wall. The right panel of Fig. 12 shows a comparison between DNS and this model. The vertical gradient of spanwise velocity is predicted within $20 \%$ accuracy. However, the maximum amplitude of the total z-component of the wall shear stress $\tau_{z}^{+}=\sum_{i=1}^{3} \sigma_{i, 3}^{+} n_{i}$ (where $\sigma_{i, 3}^{+}$are the z-components of the stress tensor at the wall and $n_{i}$ are the components of the unit vector perpendicular to the wall surface) is $50 \%$ smaller than $\frac{d w^{+}}{d y^{+}}$on the downhill slope and $25 \%$ smaller on the uphill slope. This is a result of the non-planarity of the wall and contributions from the streamwise and vertical velocity components to the spanwise shear stress. Following the calculations reported in Ref. [8], a decrease in spanwise shear stress amplitude by $50 \%$ or more with respect to the optimum value would lead to less than $1 \%$ in net power gain by the wavy wall. In Ref. [9,10], Sengupta and Lekoudis proposed a turbulent curvilinear boundary layer model for rigid or moving swept wavy walls, that accounts for the coupling of 
the wave induced shear stress and pressure gradient. It uses the Loyd model for turbulence closure of the disturbance equation system, ${ }^{17-19}$ solved consistently with the $2 \mathrm{D}$ mean flow equations. The wave geometry was similar to that studied here (with $2.54 \mathrm{~cm}$ wavelength and $h_{w} / \lambda=0.2 \%$ ), but the Reynolds number was much larger $\left(R e_{\theta}=2.25 \times 10^{6}\right)$. No net drag reduction was found for the rigid waves at any sweep angle. The power analysis of Ref. [8] could be revisited with the help of such model validated by DNS simulations, to establish whether there is a range of swept wall wave geometries and Reynolds numbers leading to some percentage of net drag and power reduction.
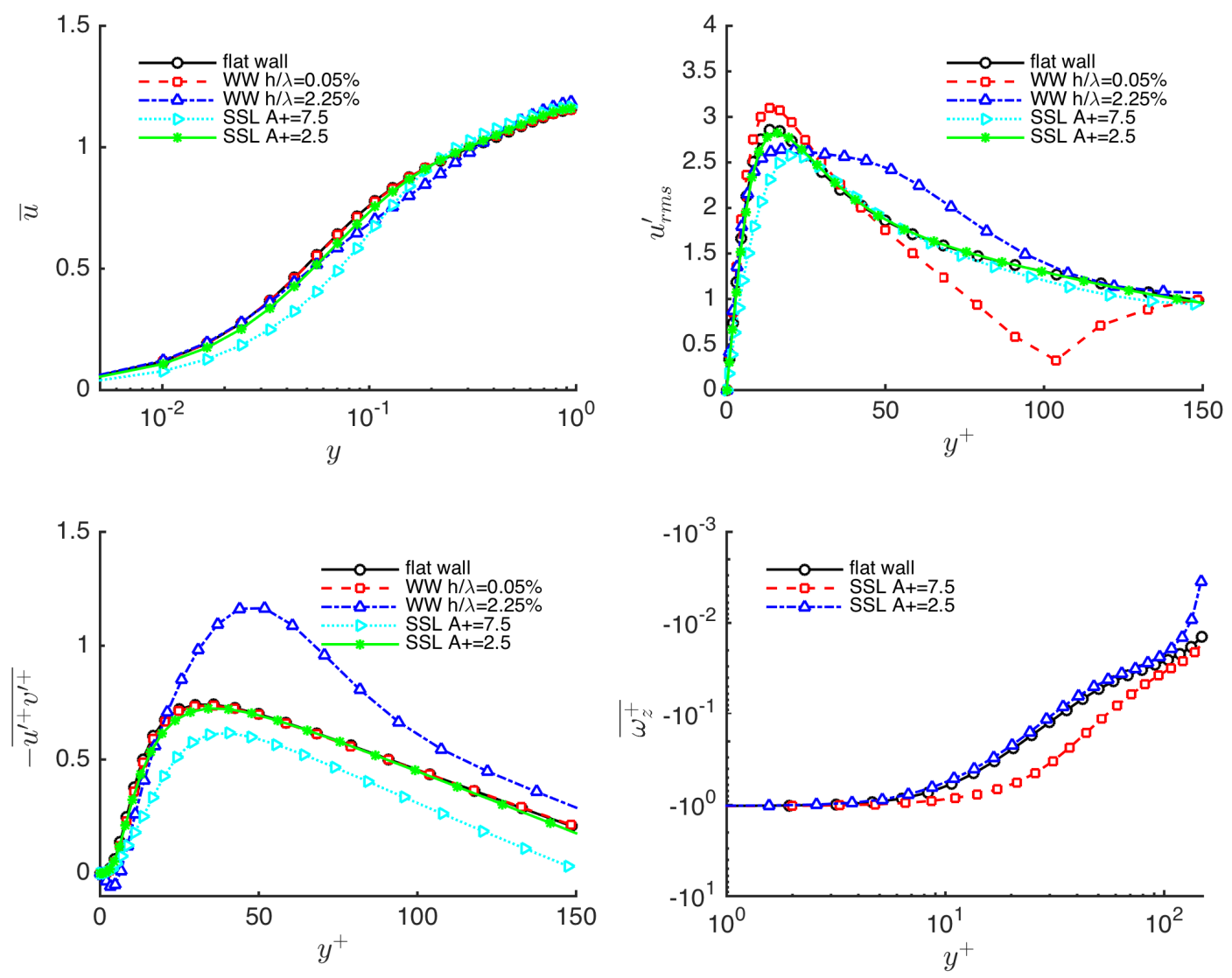

Figure 13: Time- and space-averaged profiles of streamwise velocity $\bar{u}$, streamwise velocity fluctuations $u_{r m s}^{\prime}$, Reynolds stress $-\overline{u^{\prime} v^{\prime}}$, and spanwise velocity $\overline{\omega_{z}}$.

In the following, an overline denotes a time-averaged variable $\left(\bar{f}=\int_{0}^{t \rightarrow \infty} f(t) d t\right)$ and a prime denotes the fluctuation of a quantity around its time-averaged value $\left(f^{\prime}=f(t)-\bar{f}\right)$. The term "phase-averaged" refers to the integration in both time and space over a periodic domain $\overline{f(y)}=\int_{0}^{t \rightarrow \infty} d t \int_{0}^{\lambda_{x}} d x \int_{0}^{\lambda_{z}} f(x, y, z, t) d z$. $u, v$, and $w$ are the streamwise, vertical and spanwise velocity components, respectively. Fig. 13 shows a comparison between profiles averaged over the full xz-domain $\left(2 \lambda_{x}, 2 \lambda_{z}\right)$ in the bottom half $(y<h)$ of the channel with flat wall, wavy walls, and Stokes layers. The streamwise wall shear stress, RMS of the streamwise velocity fluctuations $u_{r m s}^{\prime}=\sqrt{\overline{u^{\prime 2}}}$, and the Reynolds stress component $-\overline{u^{\prime} v^{\prime}}$ are all decreased for the SSL. The z-vorticity component for $A_{S S L}^{+}=7.65$ is decreased, as expected from the significant decrease of the shear stress at the wall. The peaks of $u_{r m s}^{\prime}$ and shear stress are shifted by about 5 wall units away from the wall, as reported in prior work. ${ }^{6}$ The phase-averaged velocity profile for the channels with wavy walls closely overlap that of the flat channel near the wall, as expected from the previous drag results. The maximum of $u_{r m s}^{\prime}$ at $y^{+}-y_{w}^{+}=15$ is $8.5 \%$ larger for the shallow waves than for the flat channel. The maximum of $u_{r m s}^{\prime}$ is $8.5 \%$ lower for the deep waves than for the flat channel, with a flat maximum extending 
into the log layer. The Reynolds stress for the deep waves peaks at $y^{+}=50$. Figures $14-15$ show turbulence statistics relative to the flat channel, along a line perpendicular to the wave crests at $y^{+}-y_{w}^{+}=15$ and along the wall. The Reynolds stress $-\overline{u^{\prime} v^{\prime}}$ is maximum on the uphill wave slope. No clear trend is seen for $u_{r m s}^{\prime}$ over the shallow waves, whereas maxima and minima are found close to $y_{w}^{+}=0$ on the positive and negative slopes of the deep waves, respectively. The wall shear stress and pressure coefficients reach their maximum value on the uphill slope, approximately three-quarters of a wavelength out of phase with the wall geometry.
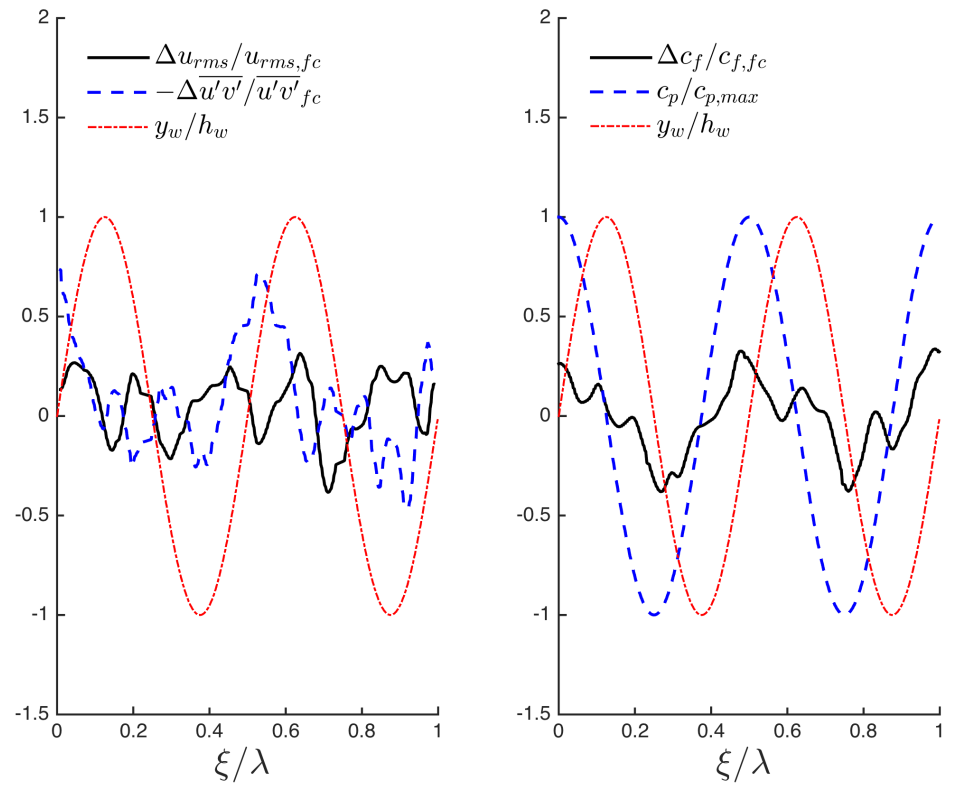

Figure 14: Deviation of $u_{r m s}^{\prime},-\overline{u^{\prime} v^{\prime}}$, and $c_{f}$ in the wavy wall with $h_{w} / \lambda=0.05 \%$, relative to the flat wall case, along a cutline perpendicular to the wave crests. The wave profile $y_{w} / h_{w}$ and pressure coefficient normalized by its maximum value $c_{p} / c_{p, \max }$ are also shown in the right panel.

\section{B. Instantaneous flow}

Fig. 16 shows the streamwise component of vorticity in a yz-plane. The x-vorticity is visibly decreased for the Stokes layer and increased for the large waves. Figures 17-18 show 3D top views of a bottom section of the channel $\left(y<h, z<\lambda_{z} / 4\right)$ at constant $u^{\prime+}=(u-\bar{u}) / u_{\tau}=-2.0$, with time steps of 5 computational units, where a computational unit is defined as $h / \sqrt{R T_{\text {ref }}}$, with $R=287 \mathrm{~J} /(\mathrm{kgK})$ and $T_{\text {ref }}=300.67 \mathrm{~K}$. The phase-averaged skin friction velocity is used for scaling. Streaks convect in the near wall region at a velocity between 8 and 12 wall units $\left(u_{\tau}\right)$, consistent with Ref. [1]. The SSL shows reduced streak density compared to the flat channel. The streaks for the shallow wall waves show similarities to the flat channel in terms of their large scale dimension and density, with a higher density of small-scale structures than in the flat channel. Such structures can also be seen for the deep waves. One might speculate whether they partly result from the convection of streaks through regions of different average velocity profiles while passing over wall troughs and crests.

In order to gain insights into the flow dynamics, we examine next the evolution of instantaneous streamwise velocity profiles around events of peak velocity fluctuation in the buffer zone. Such events are detected using the following criterion for the localized variance $\widehat{\operatorname{var}}(\mathbf{x}, t, T)$, proposed by Blackwelder and Kaplan in Ref. [21]

$$
\widehat{\operatorname{var}}(\mathbf{x}, t, T)=\frac{1}{T} \int_{t-\frac{T}{2}}^{t+\frac{T}{2}} u^{2}(\mathbf{x}, s) d s-\left(\frac{1}{T} \int_{t-\frac{T}{2}}^{t+\frac{T}{2}} u(\mathbf{x}, s) d s\right)^{2}>k_{t h} u_{r m s}^{\prime 2}
$$

where $k_{t h}$ is a threshold parameter, for example 1.2. $T$ is the averaging time for this Variable-Interval- 

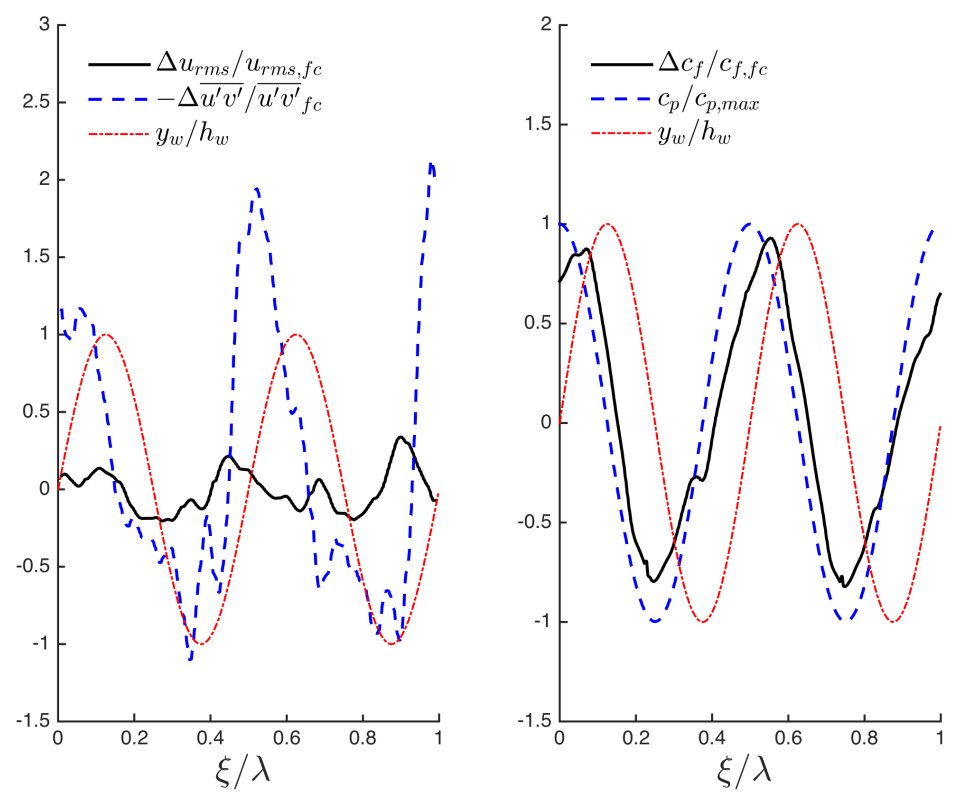

Figure 15: Deviation of $u_{r m s}^{\prime},-\overline{u^{\prime} v^{\prime}}$, and $c_{f}$ in the wavy wall with $h_{w} / \lambda=2.25 \%$, relative to the flat wall case, along a cutline perpendicular to the wave crests. The wave profile $y_{w} / h_{w}$ and pressure coefficient normalized by its maximum value $c_{p} / c_{p, \max }$ are also shown in the right panel.

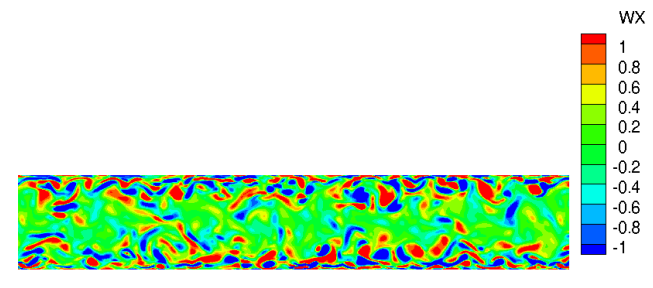

(a) Flat channel

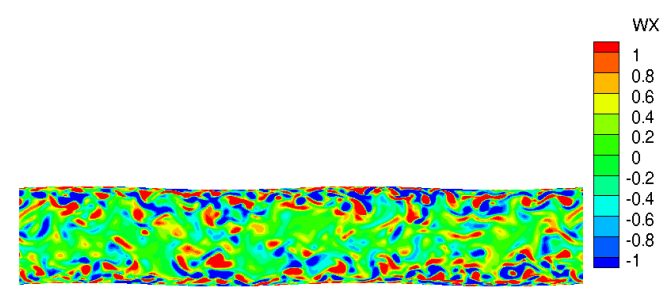

(c) $h / \lambda=0.5 \%$

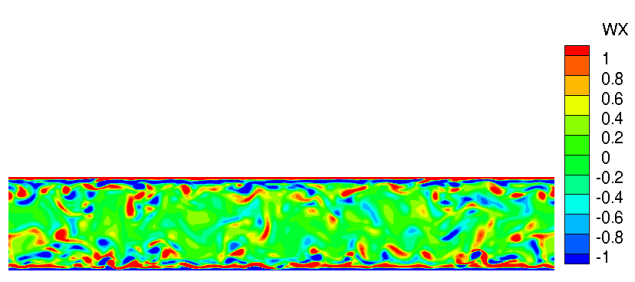

(b) Spatial Stokes layer

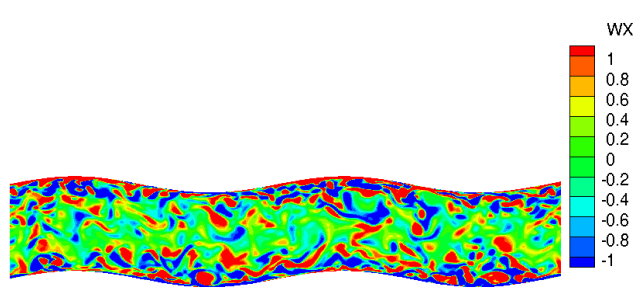

(d) $h / \lambda=2.25 \%$

Figure 16: Instantaneous streamwise vorticity in the plane defined by $x=L_{x} / 24$. 


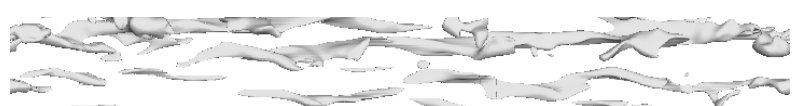

(a) $t_{0}-15, t_{0}^{+}-60.6$.

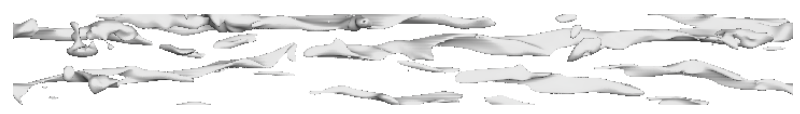

(c) $t_{0}-10, t_{0}^{+}-40.4$.

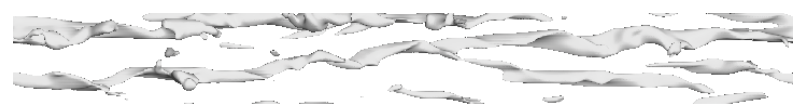

(e) $t_{0}-5, t_{0}^{+}-20.2$.

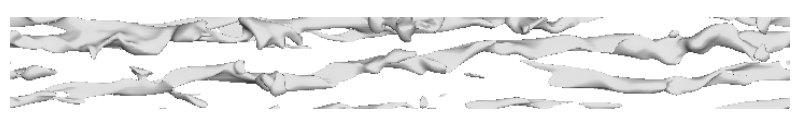

(g) $t_{0}, t_{0}^{+}$.

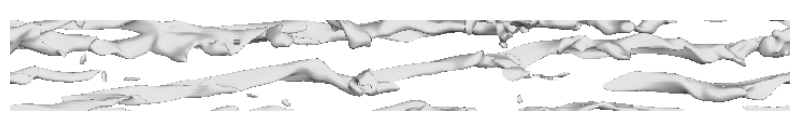

(i) $t_{0}+5, t_{0}^{+}+20.2$.

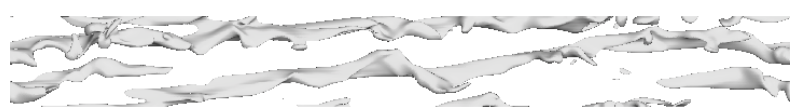

(k) $t_{0}+10, t_{0}^{+}+40.4$.

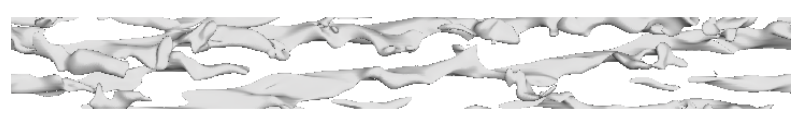

(m) $t_{0}+15, t_{0}^{+}+60.6$.

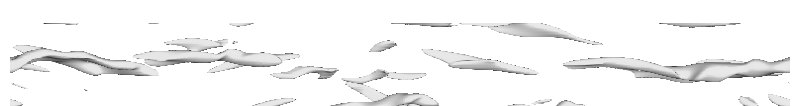

(b) $t_{0}-15, t_{0}^{+}-39.5$.

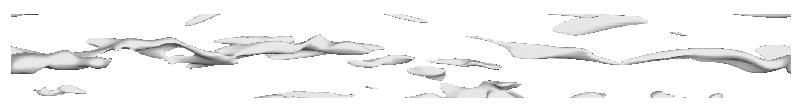

(d) $t_{0}-10, t_{0}^{+}-26.3$.

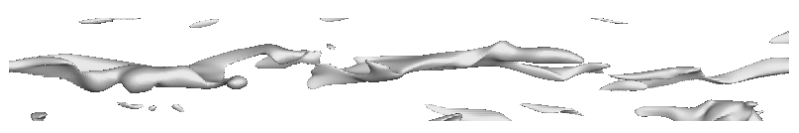

(f) $t_{0}-5, t_{0}^{+}-13.2$.

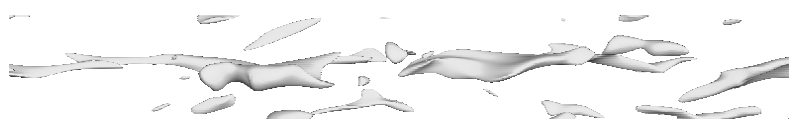

(h) $t_{0}, t_{0}^{+}$.
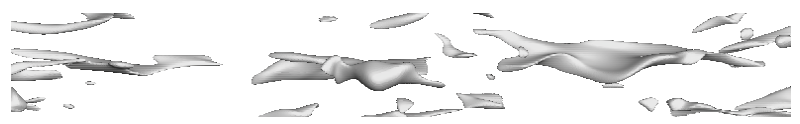

(j) $t_{0}+5, t_{0}^{+}+3.2$.
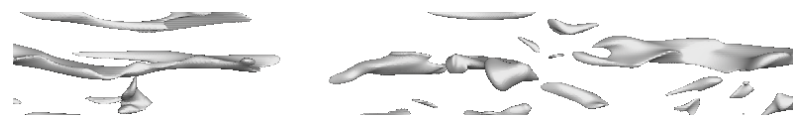

(l) $t_{0}+10, t_{0}^{+}+26.3$.

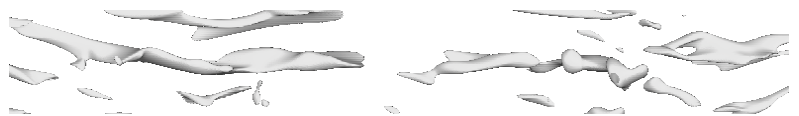

(n) $t_{0}+15, t_{0}^{+}+39.5$.

Figure 17: Top view of isocontours of stream wise velocity perturbation $u^{\prime+}=-2.0$ for the flat channel (LHS) and the SSL with $A_{S S L}^{+}=7.65$ (RHS). The flow goes from left to right. 


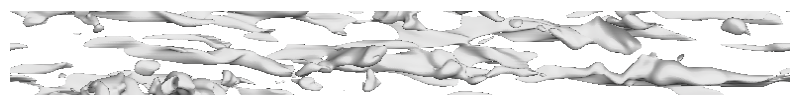

(a) $t_{0}-15, t_{0}^{+}-63.8$.

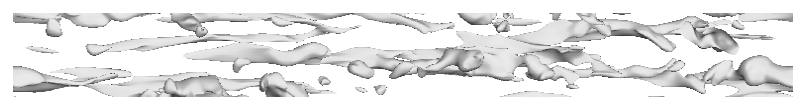

(c) $t_{0}-10, t_{0}^{+}-42.5$.

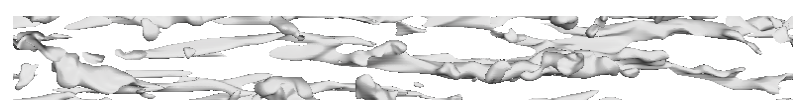

(e) $t_{0}-5, t_{0}^{+}-21.3$.

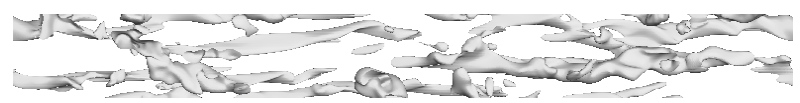

(g) $t_{0}, t_{0}^{+}$.

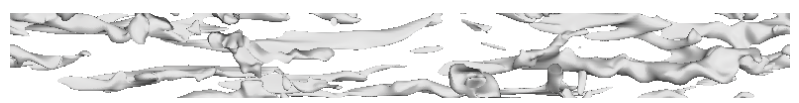

(i) $t_{0}+5, t_{0}^{+}+21.3$.

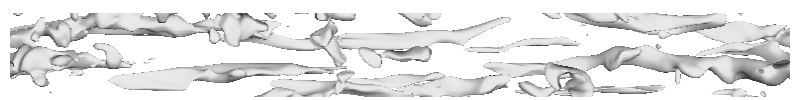

(k) $t_{0}+10, t_{0}^{+}+42.5$.

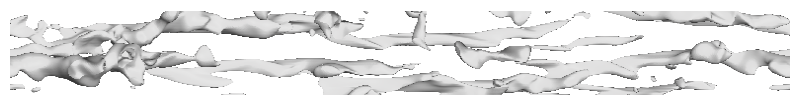

(m) $t_{0}+15, t_{0}^{+}+63.8$.

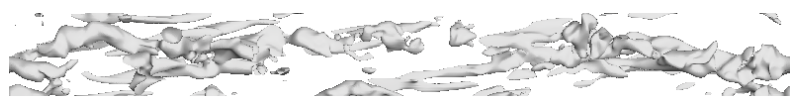

(b) $t_{0}-15, t_{0}^{+}-86.18$.

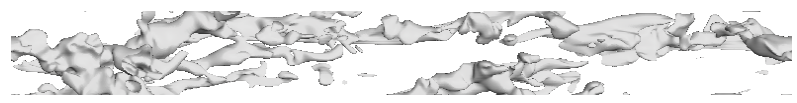

(d) $t_{0}-10, t_{0}^{+}-57.45$.

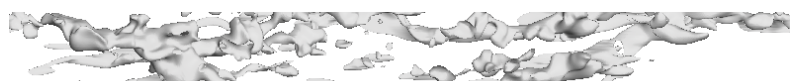

(f) $t_{0}-5, t_{0}^{+}-28.73$.

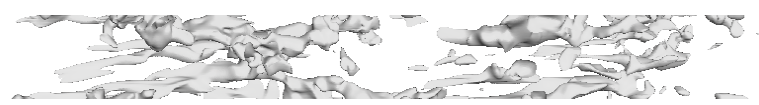

(h) $t_{0}, t_{0}^{+}$.

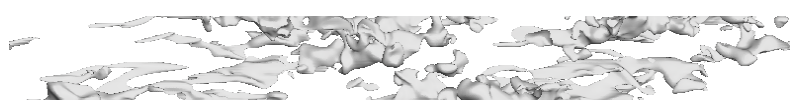

(j) $t_{0}+5, t_{0}^{+}+3.2$.

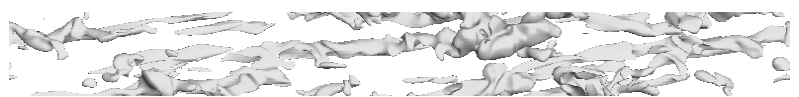

(l) $t_{0}+10, t_{0}^{+}+47.45$.

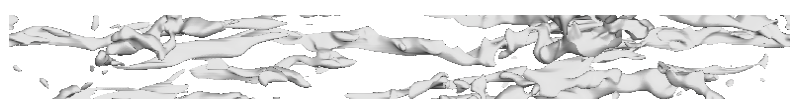

(n) $t_{0}+15, t_{0}^{+}+86.18$.

Figure 18: Top view of isocontours of stream wise velocity perturbation $u^{\prime+}=-2.0$ for the wavy wall with $h_{w} / \lambda=0.05 \%$ (LHS) and the wavy wall with $h_{w} / \lambda=2.25 \%$ (RHS). The flow goes from left to right. 
Time-Average (VITA) calculation. We use here the same value $T^{+}=10$ as in Ref. [21], where again the ' + ' subscript indicates wall units $\left(=\nu / u_{\tau}^{2}\right.$ for time). In the limit of $T \rightarrow \infty, \widehat{\operatorname{var}}(\mathbf{x}, t, T)=u_{r m s}^{\prime 2}$. When an event is detected per (1), the detection function $D\left(t^{+}\right)=1.0$. It is equal to zero otherwise. The detection coordinate is $y^{+}=15$ for all cases, except for the large Stokes waves for which we use $y^{+}=20$, according to the 5 wall unit shift of the peak of $u_{r m s}^{\prime}$ observed in Fig. 13. It is worth noting that the phase-average of $u_{r m s}^{\prime 2}$ was used for detection, instead of the $(\mathrm{x}, \mathrm{z})$-dependent time average. This allows avoiding multiple detections from a low local average value, as can arise for the wavy wall (see Figures 14-15).

Fig. 19 shows the temporal evolution of the streamwise velocity perturbation $u^{\prime}$ and localized variance $\widehat{\operatorname{var}}\left(\mathbf{x}, t^{+}, T^{+}\right)$at sampled locations within the domain of Figures $17-18$, where events with $D=1$ were detected. The reference time $t_{0}^{+}$for each case was set to the time of peak variance $\widehat{\operatorname{var}}(\mathbf{x}, t, T)$. It is also the reference time used in Figures 17-18. A spacing between events of the order of a few hundred wall units is consistent with the experimental results of Ref. [21]. The streamwise velocity fluctuation amplitude for the spatial Stokes layer with spanwise velocity forcing $A_{S S L}^{+}=7.65$ is noticeably lower than in the other cases. No clear difference is identified between the flat channel and the shallow waves. The deep waves show multiple peaks of localized variance close to the detection threshold. Figures 20-23 show the instantaneous and average streamwise velocity for each channel, at times close to the peak of the localized variance. Ideally, such profiles would result from averaging over multiple events, using conditional averaging as described in Ref. [21]. Given the computational space allocation and time requirements for such an analysis, only instantaneous profiles are plotted here, bearing in mind that they represent isolated snap-shots. The detected events show an inflectional transition from a streamwise momentum defect to a momentum excess, followed by an increase of the wall shear stress, and subsequently a recovery toward the average velocity profile. Once again, the SSL shows reduced fluctuations around the average profile and no obvious difference is observed between the flat channel and the shallow wave cases. The large waves show a large increase in wall shear stress at $t_{0}^{+}+5$ and somewhat irregular profile slope changes.

\section{Conclusions}

In this work, the turbulent drag over wall waves slanted at an angle from the streamwise direction were studied by experiments and Direct Numerical Simulations. Test plates were rapid-prototyped with two wave amplitude-to-wavelength ratios of $0.05 \%$ and $2.25 \%$, at a wave crest angle of $37.4^{\circ}$ with respect to the streamwise direction. They were measured in the NASA LaRC 7x11 Inch Low Speed Wind Tunnel (7x11) at a unit Reynolds number $0.6 \times 10^{6}<R e / m<2 \times 10^{6}$. The drag force measurement resolution for the set-up was estimated as $+/-2 \%$. Within this measurement accuracy, no drag improvement or degradation could be determined on these models relative to the flat plate. Oil vapor flow measurements showed that the flow over the waves with $h / \lambda=0.05 \%$ is parallel to the inflow, whereas the mean flow over the deep waves shows large oscillations with a mean spanwise component at an angle of about $2.5^{\circ}$ from the inflow.

The turbulent flow in channels with flat walls, swept wavy walls and spatial Stokes spanwise velocity forcing was simulated at a friction Reynolds number of two hundred by DNS. The phase-averaged and instantaneous turbulent flow characteristics of the shallow wavy wall with $0.05 \%$ amplitude-to-wavelength aspect ratio were found to compare to those of the flat channel near the wall. The friction drag is maximum on the uphill slope of the waves and minimum on the downhill slope. The pressure coefficient is out of phase with the wave profile by a quarter of a wavelength. The total drag in the channel with shallow waves was computed as $1.4 \%$ larger than in the flat channel, within the repeatability limit of the measurements. Simulation results were presented for the deep waves, however the assumed spanwise periodic boundary conditions need further consideration based on the observed mean spanwise flow. The DNS results for the Spatial Stokes layer were consistent with prior work in terms of drag reduction, turbulence statistics and linear model predictions. The linear model based on the spatial Stokes layer analogy predicted the amplitude of the vertical gradient of the spanwise velocity over the wavy wall within $20 \%$, but it was found that the total spanwise shear stress is at least twice smaller, so that the model does not provide an accurate estimate of the net power gain. Existing linear models accounting for the wave induced shear stress and mean flow modification could be used in combination with DNS to enhance prior net drag and power calculations over an extended range of swept wave geometries. 

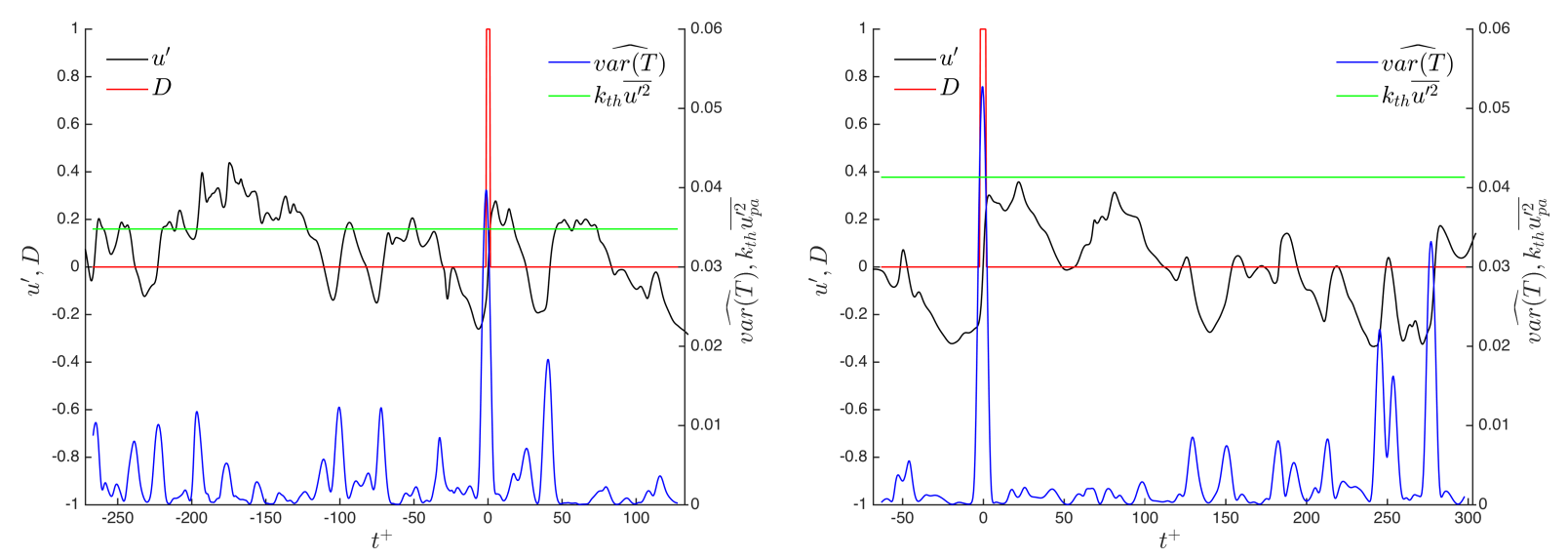

(a) Flat channel at the location $x=0.75 \lambda_{x}, z=0.12 \lambda_{z}$.

(b) Wavy wall with $h_{w} / \lambda=0.05 \%$ at the location $x=$ $0.92 \lambda_{x}, z=0.12 \lambda_{z}$.
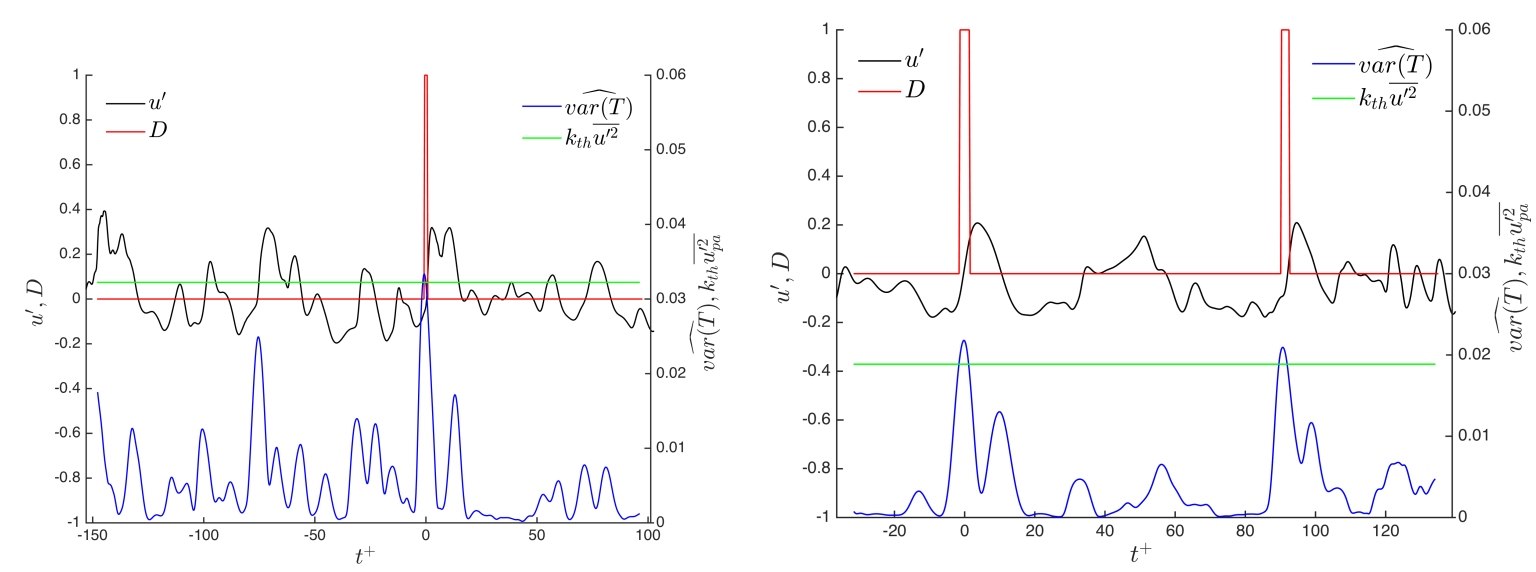

(c) Wavy wall with $h_{w} / \lambda=2.25 \%$ at the location $x=$ (d) SSL with $A_{S S L}^{+}=7.65$ at the location $x=0.75 \lambda_{x}, z=$ $0.5 \lambda_{x}, z=0.12 \lambda_{z}$. $0.12 \lambda_{z}$.

Figure 19: Time evolution of the velocity and localized variance fluctuations, along with the detection threshold and function. 


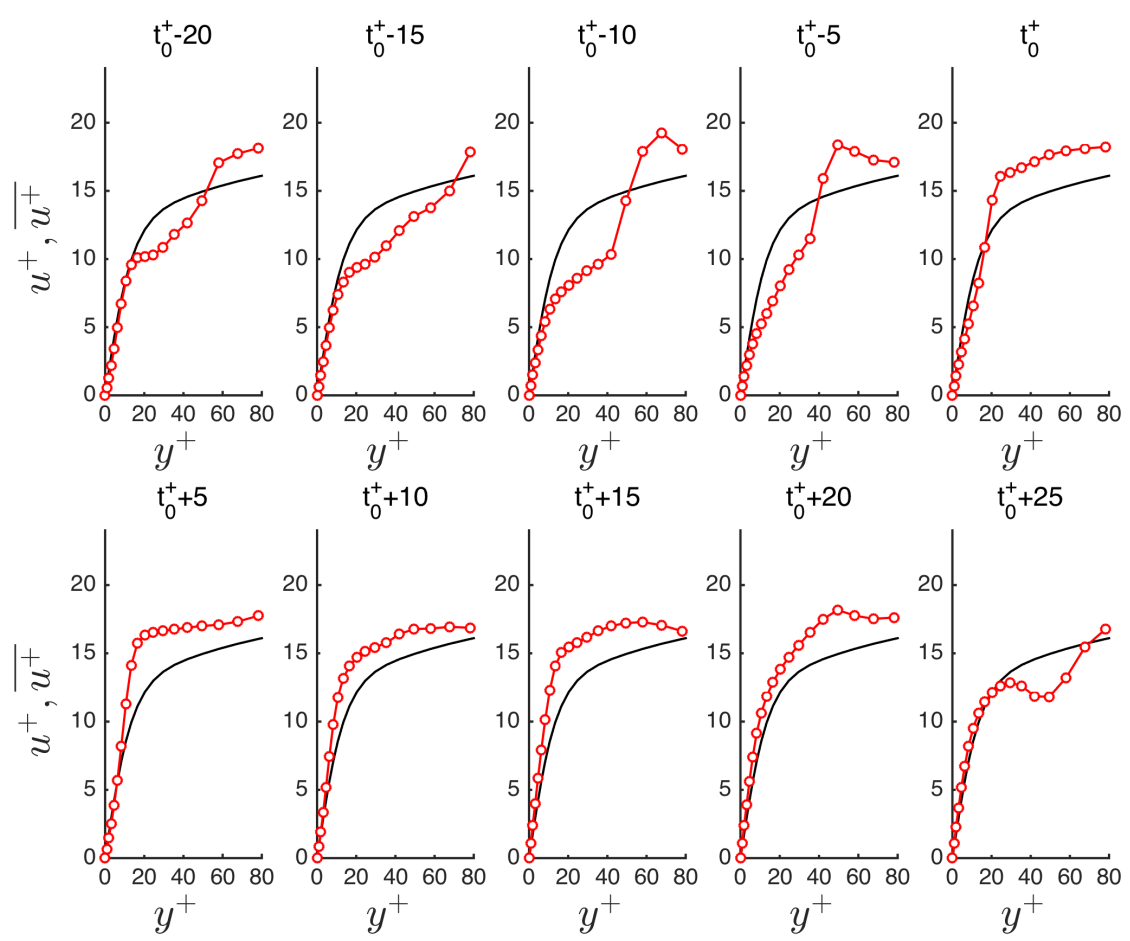

Figure 20: Streamwise velocity in the flat channel at the location $(x, z)=\left(0.75 \lambda_{x}, 0.12 \lambda_{z}\right)$. Red line and symbols: instantaneous value; black line: time average.

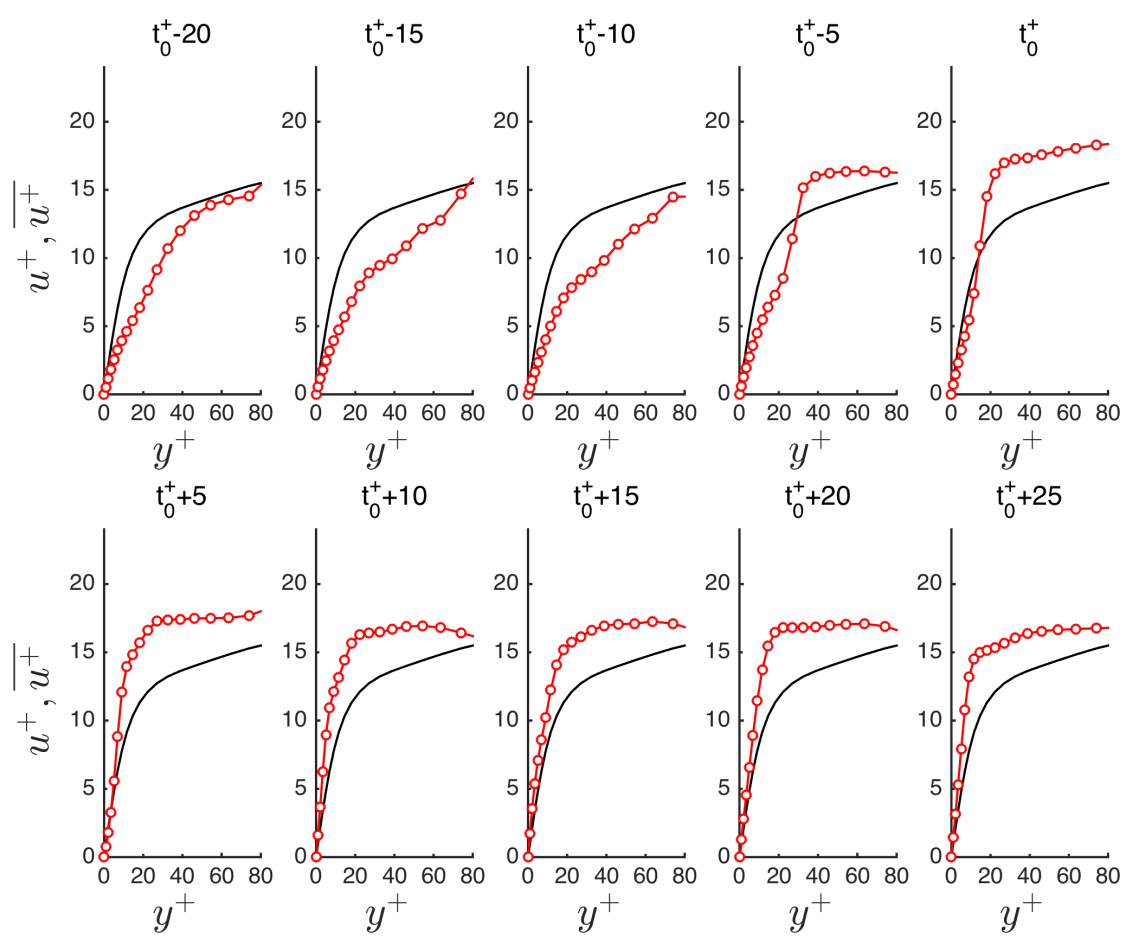

Figure 21: Streamwise velocity in the channel with wavy wall with $h_{w} / \lambda=0.05 \%$ at the location $(x, z)=$ $\left(0.92 \lambda_{x}, 0.12 \lambda_{z}\right)$. Red line and symbols: instantaneous value; black line: time average. 

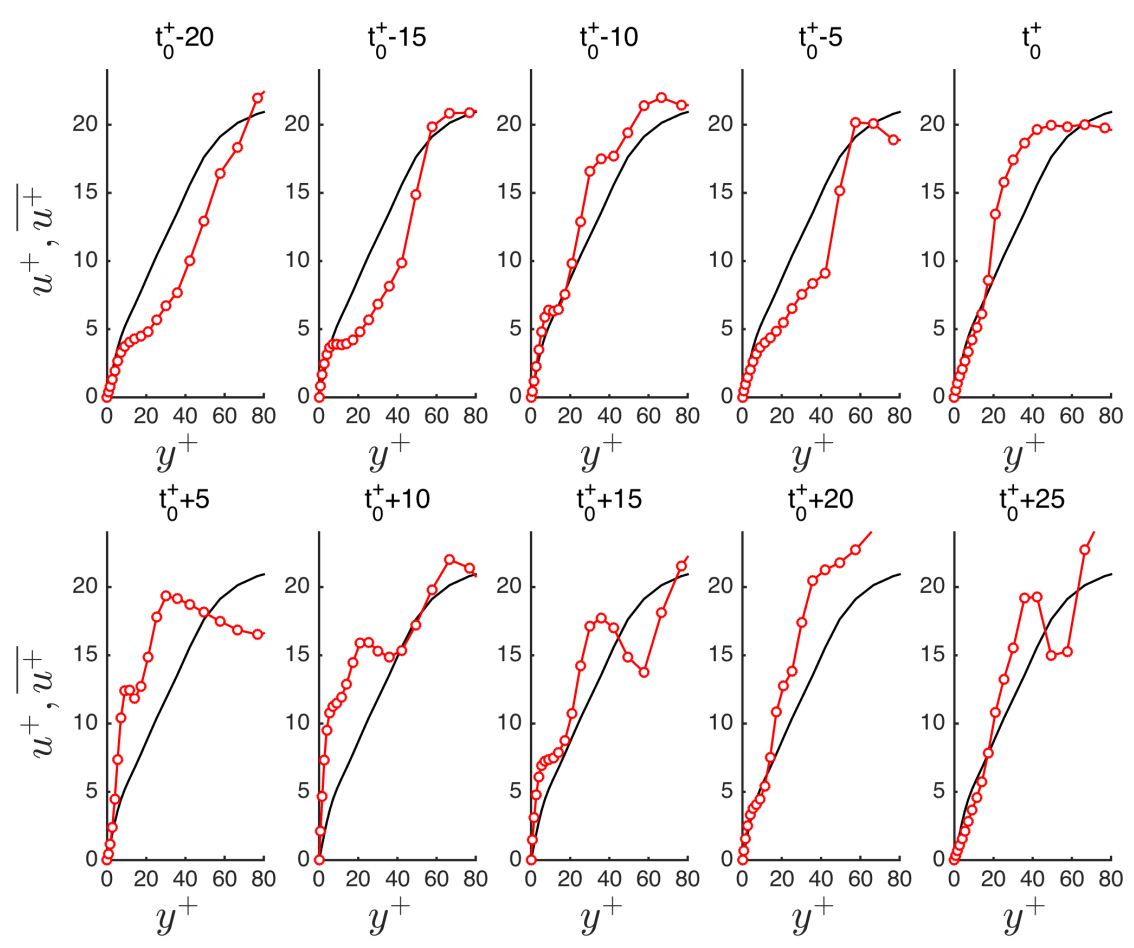

Figure 22: Streamwise velocity in the channel with wavy wall with $h_{w} / \lambda=2.25 \%$ at the location $(x, z)=$ $\left(0.5 \lambda_{x}, 0.12 \lambda_{z}\right)$. Red line and symbols: instantaneous value; black line: time average.
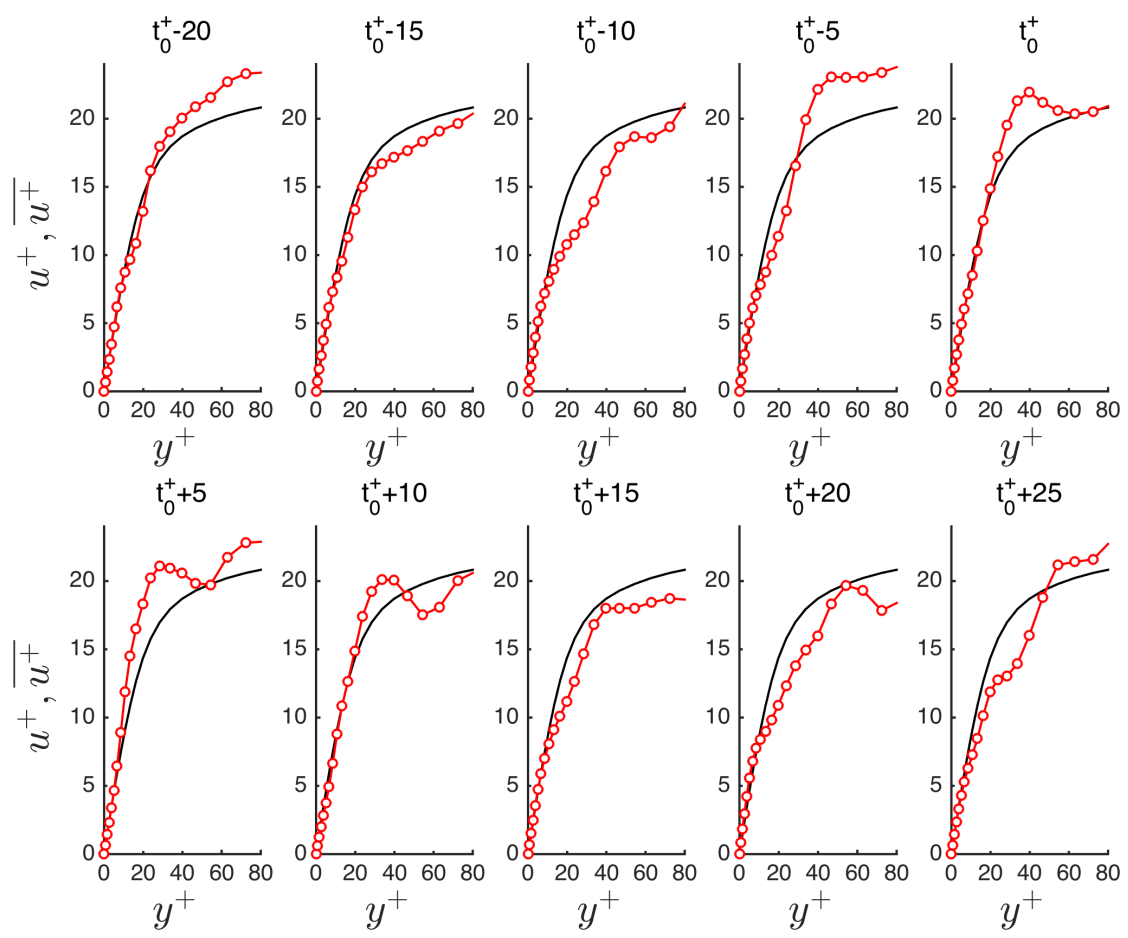

Figure 23: Streamwise velocity in the SSL channel with $A_{S S L}^{+}=7.65$, at the location $(x, z)=\left(0.75 \lambda_{x}, 0.12 \lambda_{z}\right)$. Red line and symbols: instantaneous value; black line: time average. 


\section{References}

${ }^{1}$ Kim, J. and Hussain, F., "Propagation velocity of perturbations in turbulent channel flow," Journal of Fluids A: Fluid Dynamics, Vol. 5, 1993, pp. 695-705.

${ }^{2}$ Choi, J.-I., Xu, C., and Sung, H., "Drag reduction by spanwise wall oscillation in wall-bounded turbulent flows," $A I A A$ Journal, Vol. 40, No. 5, 2002, pp. 842-850.

${ }^{3}$ Quadrio, M., Ricco, P., and Viotti, C., "Streamwise-travelling waves of spanwise wall velocity for turbulent drag reduction," J. Fluid Mechanics, Vol. 627, 2009, pp. 161-178.

${ }^{4}$ Auteri, F., Baron, A., Belan, M., Campanardi, G., and Quadrio, M., "Experimental assessment of drag reduction by traveling waves in a turbulent pipe flow," Physics of Fluids, Vol. 22, 2010, pp. 115103 1-14.

${ }^{5}$ Quadrio, M., "Drag reduction in turbulent boundary layers by in-plane wall motion," Phil. Trans. R. Soc. A, Vol. 369, 2011, pp. 1428-1442.

${ }^{6}$ Viotti, C., Quadrio, M., and Luchini, P., "Streamwise oscillation of spanwise velocity at the wall of a channel Streamwise oscillation of spanwise velocity at the wall of a channel for turbulent drag reduction," Physics of Fluids, Vol. 21, 2009, pp. 115109 $1-9$.

${ }^{7}$ Skote, M., "Comparison between spatial and temporal wall oscillations in turbulent boundary layer flows," J. Fluid Mechanics, Vol. 730, 2013, pp. 273-294.

${ }^{8}$ Chernyshenko, S., "Drag reduction by a solid wall emulating spanwise oscillations. Part 1." Phys. Fluid Dyn., 2013.

${ }^{9}$ Sengupta, T., Turbulent boundary layers over rigid and moving wavy surfaces, Ph.D. thesis, Georgia Institute of Technology, June 1984.

${ }^{10}$ Lekoudis, S. and Sengupta, T., "Two-dimensional turbulent boundary layers over rigid and moving swept wavy surfaces," Physics of Fluids, Vol. 29, 1986, pp. 964-970.

${ }^{11} \mathrm{Shu}, \mathrm{C} . \mathrm{W} .$, "Essentially non-oscillatory and weighted essentially non-oscillatory schemes for hyperbolic conservation laws," Tech. Rep. NASA/CR-97-206253, ICASE Report No. 97-65, NASA, 1997.

12 Atkins, H., "High-order ENO methods for the unsteady compressible Navier-Stokes equations," No. AIAA-91-1557, 1991.

${ }^{13}$ Balakumar, P., "Receptivity of a supersonic boundary layer to acoustic disturbances," AIAA Journal, Vol. 47, No. 5, 2009, pp. 1034-1041.

${ }^{14}$ Roberts, G., "Computational meshes for boundary layer problems," Proc. Second Int. Conf. Num. Methods Fluid Dyn., edited by S. Verlag, Vol. 8, Computational meshes for boundary layer problems, New York, 1971, pp. 171-177.

${ }^{15}$ Wilcox, D., Turbulence modeling for CFD, DCW Industries, third edition ed., 2010.

${ }^{16}$ Quadrio, M. and Ricco, P., "Critical assessment of turbulent drag reduction through spanwise wall oscillations," J. Fluid Mechanics, Vol. 521, 2004, pp. 251-271.

${ }^{17}$ Thorsness, C., Transport phenomena associated with flow over a solid wavy surface, Ph.D. thesis, University of Illinois, 1975.

${ }^{18}$ Cary, A., Weinstein, L., and Buschnell, D., "Drag reduction characteristics of small amplitude rigid surface waves," Progress in Astronautics and Aeronautics: Viscous Flow Drag Reduction, 1980, pp. 144-167.

${ }^{19}$ Thorsness, C., Morrisroe, P., and Hanratty, T., "A comparison of linear theory with measurements of the variation of shear stress along a solid wave," Chem. Eng. Science, Vol. 33, 1978, pp. 579-592.

${ }^{20}$ Schoppa, W. and Hussain, F., "Coherent structure generation in near-wall turbulence," J. Fluid Mechanics, Vol. 453, 2002, pp. 57-108.

${ }^{21}$ Blackwelder, R. and Kaplan, R., "On the wall structure of the turbulent boundary layer," J. Fluid Mechanics, Vol. 76, part 1, 1976, pp. 89-112. 